\title{
Efficient encapsulation of large theragnostic nanoparticles in cell-derived exosomes: Leveraging the exosomal biogenesis pathway to obtain hollow gold nanoparticle-exosome hybrids
}

\author{
María Sancho-Albero ${ }^{a, b}$, Dr. Marimar Encabo-Berzosa ${ }^{a, b}$, Manuel Beltrán-Visiedo $^{a, b}$, Dr. Lola \\ Fernández-Messina, ${ }^{c, d}$, Dr. Víctor Sebastián ${ }^{a, b}$, Prof. Francisco Sánchez-Madrid ${ }^{c, d}$, Dr. Manuel \\ Arruebo $^{a, b, *}$, Prof. Jesús Santamaria, ${ }^{a, b, *}$ and Dr. Pilar Martín-Duque $e^{e, f}$
}

a Department of Chemical Engineering, Aragón Institute of Nanoscience (INA). Univeristy of Zaragoza, Campus Rio Ebro-Edificio I+D+I, C/ Mariano Esquillor S/N, 50018- Zaragoza-Spain.

b Networking Research Center of Bioengineering, biomaterials and Nanomedicine, CIBER-BBN, 28029Madrid, Spain

c Servicio de Inmunología, Instituto de Investigación Sanitaria Princesa (IP), Hospital Universitario de la Princesa, Universidad Autónoma de Madrid. C/ Diego de León 62, 28006-Madrid, Spain.

d Centro de Investigación Biomédica en Red de Enfermedades Cardiovasculares (CIBER-CV), Av. Monforte de Lemos 3-5, 28029-Madrid, Spain. e Fundación Araid, 500009-Zaragoza, Spain.

f Instituto Aragonés de Ciencias de la Salud (IACS/IICS Aragón), Centro de Investigación Biomédica de Aargón (CIBA), Zaragoza, Spain.

\section{Corresponding author}

Dr Manuel Arruebo and Prof. Jesús Santamaría

Department of Chemical Engineering

Aragon Nanoscience Institute

C/Mariano Esquillor s/n

Zaragoza University

50018 Zaragoza, Spain

Tel: $+34876555437+3487655545311$

Email: arruebom@unizar.es, jesus.santamaria@unizar.es 


\section{$\underline{\text { Abstract }}$}

Exosomes are considered as naturally targeted delivery systems able to carry exogenous payloads, drugs or theranostic nanoparticles (NPs). This work aims to combine the therapeutic capabilities of hollow gold nanoparticles (HGNs) with the unique tumor targeting properties provided by exosomes. Here, we tested different methods to encapsulate HGNs (capable of absorbing light in the NIR region for selective thermal ablation) into murine melanoma cells derived exosomes (B16-F10-exos), including electroporation, passive loading by diffusion, thermal shock, sonication and saponin-assisted loading. These methods gave less than satisfactory results: although internalization of relatively large NPs into B16-F10-exos was achieved by almost all the physicochemical methods tested, only about $15 \%$ of the exosomes were loaded with NPs and several of those processes had a negative effect in the morphology and integrity of the loaded exosomes. In a different approach, B16-F10 cells were pre-incubated with PEGylated HGNs (PEG-HGNs) in an attempt to incorporate the NPs into the exosomal biogenesis pathway. The results were highly successful: exosomes recovered from the supernatant of the cell culture showed up to $50 \%$ of HGN internalization. The obtained hybrid HGN-exosome vectors were characterized with a battery of techniques to make sure that internalization of HGNs did not affect exosome characteristics compared with other strategies. PEG-HGNs were released through the endosomal-exosome biogenesis pathway confirming that the isolated vesicles were exosomes.

\section{Background}

Gold-based NPs are one of the workhorses in Nanomedicine, with applications in widely different fields including biosensing,1 drug delivery,2 near-infrared (NIR)-based hyperthermia,3 imaging 4 and bio-orthogonal catalysis.5 Gold is a suitable candidate for 
those applications due to its low toxicity at the studied doses, inertness and biocompatibility compared with other types of materials. 6 In addition, nanogold has a wealth of interesting properties, among which the "surface plasmon resonance (SPR)" effect is outstanding.7 The SPR effect of gold nanoparticles (AuNPs) can be exploited in a wide range of approaches, including labelling of living cells, 8 imaging,9-11 diagnosis12-14 and therapy.3,15,16 In tumor therapy, a variety of strategies have been employed to selectively deliver AuNPs to tumors: i) development of an outside layer, typically PEG, that hinders the adsorption of proteins and retards detection by the immune system,17 ii) functionalization with targeting moieties (such as antibodies, aptamers or internalization peptides) 18 and iii) Trojan-Horse strategies (i.e. loading the particles inside cell types having tropism towards the desired objective).3 Nevertheless, these strategies still present unsolved challenges and the preferential accumulation of PEGylated NPs in tumors is very modest.19

Exosomes are vesicles of endocytic origin from 20 to $200 \mathrm{~nm}$ in diameter secreted by the majority of the cells present in the organism. They are surrounded by a lipidic membrane similar to the plasmatic membrane enriched in lipids such as sphingomyelin, phosphatidylserine, phosphatidylcholine, phosphatidylethanolamine, ceramide and cholesterol.20 Exosomes also contain cytosolic components from the parental cell, including mRNA, miRNA, DNA and proteins, which can be horizontally transferred to recipient cells.21 These nanovesicles are characterized by the presence of specific proteins, independently of the parental cell. In particular, it is well know that the exosome membrane contains proteins from a variety of families such as tetraspanines (CD63, CD81 or CD9), heat shock proteins (Hsp70), lysosomal proteins (Lamp2b), molecules involved in multivesicular bodies (MVBs) biogenesis such as TSG101 and Alix or fusion proteins such as flotilin or anexin.22 Exosomes formation is a multistep process that includes i) formation of intraluminal vesicles (ILVs) in MVBs, ii) transport of MVBs to the plasma membrane, 
and iii) fusion of MVBs with the cellular membrane.23 Until recently, the primary role attributed to exosomes was the elimination of unnecessary or toxic material from cells. However, today exosomes are considered as natural signalling vectors that play an essential role in intercellular communication processes and in the transfer of small molecules among cells. 24 Moreover, they have been proposed to be crucial in cancer progression pathways (evasion of the immune system, generation of pro-tumoral niches, promotion of angiogenesis and tumoral cell proliferation), 25 in the development of the nervous system,26 in neurodegenerative diseases of the brain 27 and in cardiovascular diseases. 28 Furthermore, it is known that exosomes secrete specific molecules to receptor cells and that they can reach different target tissues according to the characteristics of the cells from which they were originated, endowing them with exciting possibilities regarding selective targeting.29 In view of the above, but especially taking into account the potential targeting capabilities of exosomes, it is not surprising that exosomes are emerging as ideal delivery vehicles for theranostic applications. A number of attempts have been made to introduce a therapeutic payload within exosomes. A decade ago, small lipophilic molecules were passively introduced in exosomes by incubation at room temperature.30 Then, more complex molecules including anti-oxidants and anti-inflammatory compounds were loaded inside them.31-36 The loading of NPs within exosomes is even more challenging due to the large dimensions (compared to molecules) and the solid nature of the particles. This means that, simple and diffusion-driven methods that rely on the simultaneous incubation of NPs with exosomes are hardly used. However, loading NPs into exosomes is highly desirable because it combines the attractive features of exosomes as delivery vectors with the unique properties of NPs as signalling or therapeutic agents. Therefore, more aggressive physical methods have been tested, although still with few successful results. Most of the published works have addressed the loading of magnetic NPs within exosomes to provide them with multiple 
capabilities as contrast agents in magnetic resonance imaging (MRI) while serving as drug vehicles.37-41 Hood et al., optimized an electroporation procedure to load $5 \mathrm{~nm}$ superparamagnetic iron oxide nanoparticles (SPIONs) in exosomes while minimizing their aggregation.37 A similar strategy was used by $\mathrm{Hu}$ et al. to accumulate exosomes loaded with SPIONs in the lymphatic nodes using magnetic NPs of $5 \mathrm{~nm}$ as contrast agents in MRI.38 Recently, Qi et al. reported the isolation of serum exosomes by using magnetic NPs of almost $10 \mathrm{~nm}$ functionalized with transferrin.41 In this case the NPs were attached to the external surface of exosomes, rather than being loaded inside them. Even fewer studies combine exosomes with AuNPs. In 2014, Alhasan et al. incorporated AuNPs of 13 nm of diameter conjugated with anti-miRNA21 in human prostate PC-3 cells derived exosomes.42 Betzer et al. successfully labelled exosomes with glucose-coated AuNPs (from 5 to $20 \mathrm{~nm}$ of diameter). They were incorporated on the exosomes assisted by a glucose-dependent active mechanism using the resulted hybrid exosomes in non invasive in vivo neuroimaging.43 Last year, Roma-Rodrigues et al. functionalized AuNPs with thiolated oligonucleotides for the selective silencing of an essential gene for the biogenesis and processing of exosomes, and thus decreasing exosomal production.44 In spite of those excellent results that show the potential of combining exosomes with AuNPs, studies comparing different methodologies for an efficient encapsulation of NPs within exosomes are still lacking. Motivated by the possibility of using exosomes as tumor-selective carriers for therapeutic AuNPs (capable of absorbing light in the NIR for optical hyperthermia therapy), we have attempted the encapsulation of entities larger than most of the previously published works (namely 40-45 nm HGNs) within exosomes by several strategies. Appropriate dimensions of the Au nanostructures are key to attaining SPR in the NIR region that can be used for in vivo hyperthermia-based treatments.3,45 Herein, different physicochemical methods have been tested to load these $40 \mathrm{~nm}$ HGNs in exosomes derived from B16-F10 cells. In addition, a 
method involving the direct incubation of cells with HGNs and the subsequent exosome collection has also been tested. Remarkably, when exosomes derived from cells incubated with HGNs were purified directly from the supernatant fluid, a high yield of exosomes loaded with HGNs was obtained.

\section{$\underline{\text { Results and discussion }}$}

\section{Characterization of HGNs and B16-F10-exos}

HGNs as well as B16-F10-exos were thoroughly characterized by a battery of physicochemical (TEM, DLS and Zeta potential) and biological techniques (WB, Nanosight and BCA) detailed in the supplementary material. As previously reported results, Fig. 1 shows that NPs morphology and properties correspond with the ones described in the literature.3,45,46 B16-F10-exos properties were also as the ones reported in previous studies (Fig. 2).47,48,49,50
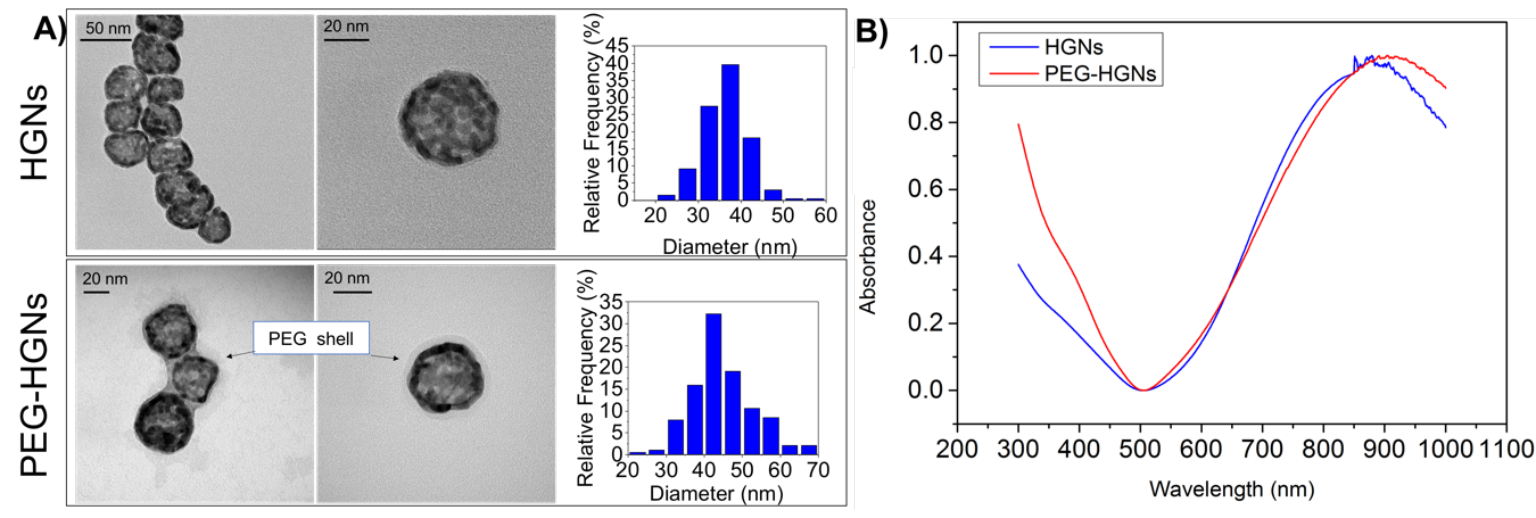

Figura 1. HGNs and PEG-HGMs characterization. A) TEM images and size distribution histograms (> 200 particles measured) of HGNs (top) and PEG-HGNs (bottom). A $5 \mathrm{~nm}$ PEG shell can be observed surrounding HGNs after PEGylation when they were stained with phosphotungstic acid. B) UV-VIS absorption spectra for both NPs dispersions. 

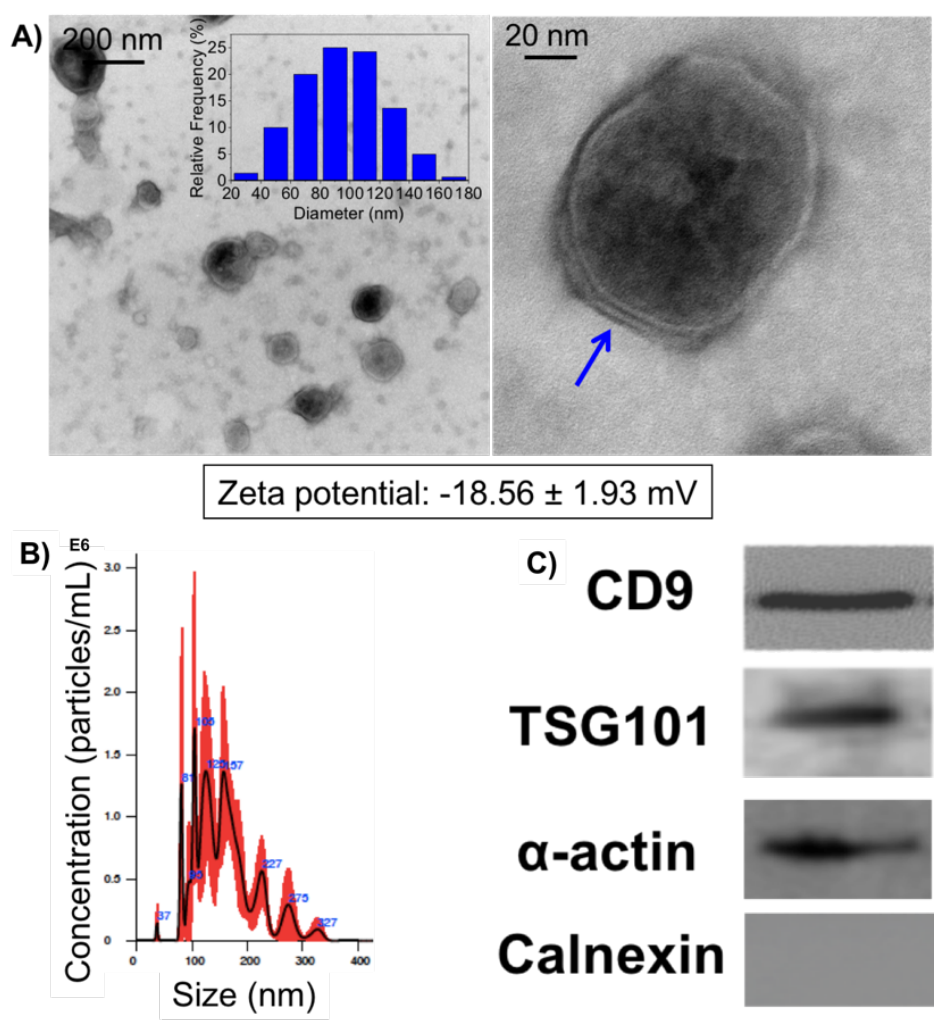

Figura 2. Characterization of B16-F10-exos. A) TEM images of isolated exosomes and size distribution histogram obtained from electronic microscopy images. Blue arrow indicates the double exosomal membrane. B) NTA measurement of exosomes. C) WB analysis showing the expression of two specific exosomal markers (CD9, TSG101) and of one housekeeping protein ( $\alpha$-actin) in exosomes. The expression of calnexin was evaluated to confirm the lack of expression of a non-exosomal protein.

\section{Physicochemical methods for HGNs loading within exosomes}

Several works reported the encapsulation of therapeutic molecules within exosomes. For instance, Zhuang et al. observed that curcumin-loaded exosomes were able to significantly reduce lipopolysaccharide-induced brain inflammation in vivo.31 In another study, Haney et al. employed ex vivo loading methods for the encapsulation of catalase in macrophage derived exosomes. The encapsulation of the enzyme within exosomes preserved its 
enzymatic activity, prolonged blood circulation time and reduced immunogenicity. 32 Herein, HGNs internalization within B16F10-exos was studied using different physicochemical methods.
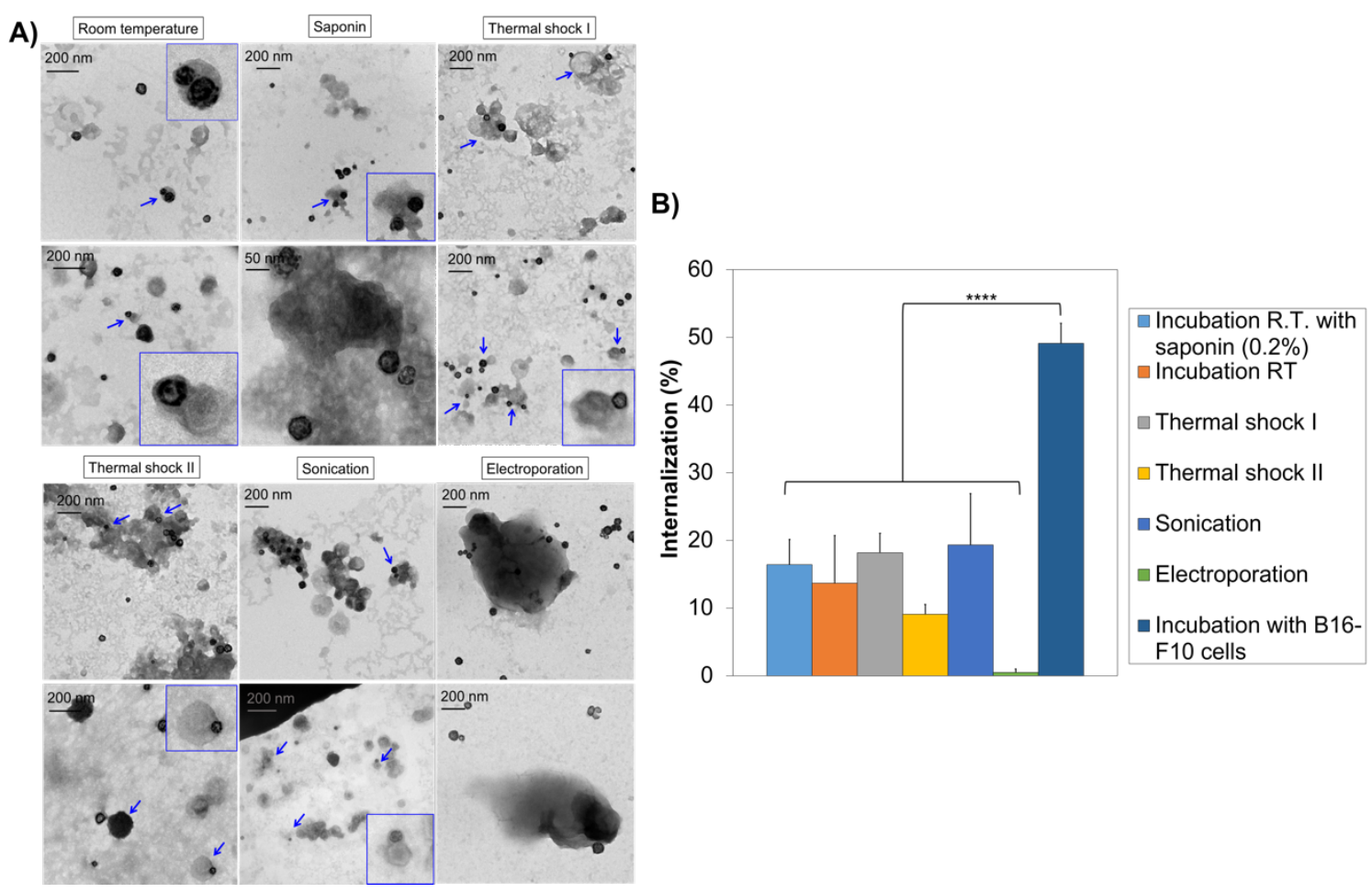

Figura. 3. A) TEM images of exosomes incubated with HGNS and encapsulated using different strategies: incubation at room temperature with the presence or absence of saponin, two different thermal shock procedures, sonication and electroporation. Blue arrows indicate exosomes loaded with HGNs. B) Internalization percentage obtained for the different methods. The amount of HGNs loaded into exosomes varied from $7 \%$ to $19 \%$ and increased as follows: electroporation $<$ thermal shock II < incubation at room temperature $<$ incubation with saponin $<$ thermal shock II < sonication $<$ Incubation with B16-F10 cells. Error bars $\pm S D, n=3 . * * * * P<0.0001$

The obtained HGNs-loaded exosomes by the different strategies were visualized by TEM and the percentage of loaded exosomes was estimated from the electronic microscopy images (Fig. 3A). The semiquantive analysis of the encapsulation efficiency achieved by the 
different approaches is shown in Fig. 3B. As the role of saponin as an effective detergent is well known,51 it was incorporated into the HGNs together with the exosome-based suspension to facilitate pore opening in the exosomal membrane favouring HGNs encapsulation. The encapsulation yield increased somewhat from $13.7 \pm 9.9 \%$ to $16.4 \pm 5.1$ $\%$ when saponin was added to the mixture compared to the situation when HGNs were allowed to passively diffuse inside exosomes. Fig. S1 indicates that neither the passive incubation of HGNs with exosomes at room temperature, nor the use of saponin affected exosome morphology. However due to the relatively low encapsulation yields achieved when saponin was added, and to the concerns regarding to its haemolytic function, the use of this permeabilizer would be limited in vivo. 52

Encapsulation yields of $18.20 \pm 1.35 \%$ and $9.11 \pm 2.0 \%$ were respectively obtained when thermal shocks I and II were applied. Differences on the incorporation yields obtained between the two thermal methods might be due to the temperatures reaches and the pore sizes obtained with them. While freezing down at $-80{ }^{\circ} \mathrm{C}$ (thermal shock I) might disrupt permanently the exosomal membrane, thermal shock II might produce smaller and temporal pores. Haney et al, already used thermal shock I to include catalase on the exosomes 32 but the size and type of compound (drug, nanoparticle etc) and the hole size would be important for their incorporation inside the vesicles. Herein, not only low encapsulation yields of HGNs were obtained but also the process affected exosomal morphology and induced aggregation. As the exosomes morphology was not affected by both thermal shock strategies in absence of NPs (Fig. S1), we attributed the exosome damage to the massive deformation that their membranes undergo when they incorporated HGNs inside their compartment following this thermal route. In fact, it is described that freeze and thaw cycles on exosomes in the presence of some drugs can induce their rupture and their aggregation.32 
Similar to whole cells, Kim et al., have shown that when suing sonication, exosome membrane viscosity significantly decreases.53 Thus, sonication could create holes/pores in the exosomal lipid bilayer promoting NPs incorporation. In fact, Haney et al., reported the internalization of catalase within exosomes using ultrasounds.32 Interestingly, our results showed that mild sonication treatment increased the loading yield compared with the previous methods $(19.34 \pm 10 \%)$ and TEM images also suggest that the effect of this process on exosome morphology was minimal (Fig. 3). In fact, it seems to be more effective than the previous methods: the loading yield obtained was the highest of the different physicochemical approaches employed. We hypothesize that when submitting exosomes to mild sonication, a reorganization and reshaping of their membrane take place, and the presence of HGNs in the vicinity leads to a large number of encapsulation events.54 Nevertheless, TEM images revealed that some NPs were attached to exosomal membrane rather than being inside them. In fact, it is reported that sonication could promote the attachment of drugs to the outer layer of membranes when the membrane reorganization process takes place. 53

Electroporation has been used for the encapsulation of nucleic acids into exosomes. For instance, Alvarez-Erviti et al. used electroporation to introduce siRNAs in exosomes for the treatment of Alzheimer disease.33 Electroporation of exosomes was also employed by Ohno et al. to load miRNA to inhibit the expression of epidermal growth factors in human breast cancer cells.34 This technique disturbs the exosomal membrane opening temporary pores in the presence of an electrical field in a conductive solution.55 In our study, electroporation seems to cause unacceptable damage to exosomes (TEM images shown in Fig. 3 of electroporated exosomes in the presence of HGNs revealed significant effects on exosomes morphology and the formation of large organic aggregates). In fact, exosome electroporation procedure by itself was extremely harmful for the exosome integrity (Fig. S1). 
In summary, with the methods reported so far, no statistical differences were obtained among mild sonication, thermal shock I, thermal shock II and incubation at RT with or without saponin. On the contrary, significant differences were observed between these approaches compared with electroporation. Electroporated exosomes in the presence of HGNs showed not only very low encapsulation yields but also significant effects on exosomes morphology and large aggregates were observed, evidencing that this method was extremely harmful for the exosome integrity. The statistical analysis is detailed in the supplementary material.

The NPs encapsulation efficiencies herein obtained were comparable (and sometimes better) than those showed in previous reports for smaller molecules and drugs. Yang et al. reported a drug loading yield of $7.2 \%$ and $11.7 \%$ for placitaxel (PTX) and doxorubicin (DOX) respectively, by incubation at room temperature.36 Also, Raposo et al. tested different procedures for the encapsulation of catalase in exosomes obtaining loading yields from 20 $\%$ to $26 \% .22$ Finally, Kim et al., incorporated PTX into exosomes and the amount of the chemotherapeutic agent increased as follows: incubation at RT $<$ electroporation $<$ sonication. 53

Due to the low encapsulation efficiencies obtained by the physicochemical approaches, we decided to use the cellular machinery to encapsulate the NPs inside exosomes. We initially tested the cytotoxicity of the PEG-HGNs in B16-F10 cells in order to obtain the HGNs subcytotoxic dose to achieve their encapsulation into exosomes following the biogenesis pathway of the cells without causing cytotoxic effects.

\section{Cytotoxicity evaluation}

The Blue Cell Viability assay (Fig. 4A) revealed that PEG-HGNs did not significantly affect cell viability at any of the studied doses in the time intervals tested. These results are in agreement with other works developed in our laboratory with the same particles.46 
Nevertheless, when B16-F10 cells were incubated with HGNs a decrease on cell viability could be observed for the highest doses $(0.5 \mathrm{mg} \mathrm{mL}-1)$. This is in contrast with PEG-HGNs treatment, whose cell viability was not significantly changed when time increased. According to the ISO 10993-5 (Biological evaluation of medical devices. Part 5: Test for in vitro cytotoxicity) a reduction in cell viability higher than $30 \%$ compared to the control is considered as a cytotoxic effect. Consequently, $0.125 \mathrm{mg} \mathrm{mL}-1$ was chosen as a subcytotoxic dose for HGNs and PEGylated HGNs for the following experiments.

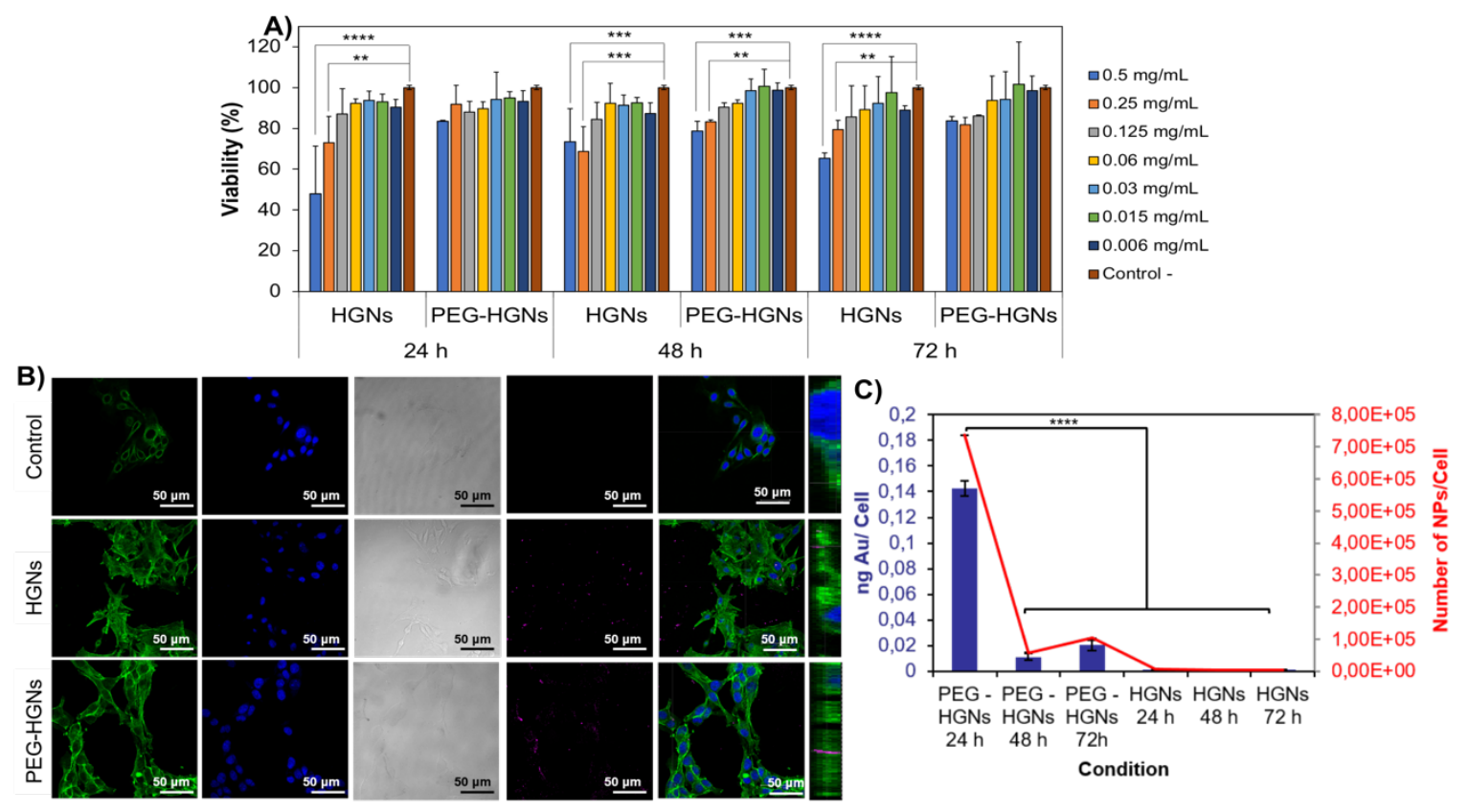

Figura 4. A) Viability of B16-F10 cells treated with HGNs and PEG-HGNs during 24, 48 and 72 h. B) Confocal images of B16-F10 cells incubated without treatment (control), with HGNs or PEG-HGNs. Green channel corresponds with actin filaments labelled with 488phaloidin, blue channel show nuclei stained with Draq5 and pink channel indicates reflection from nanoparticle agglomerates. Orthogonal projections reveal pink spots of agglomerated HGNs and PEG-HGNs inside cell cytoplasm. C) Gold content (mg/cell) obtained from by MP-AES analysis of cell cultures after removing non-internalized NPs at 24, 48 and 72 h. Error bars $\pm S D, n=3 .{ }^{* *} P<0.01, * * * P<0.001, * * * * P<0.0001$ 


\section{Incorporation of PEG-HGNs in the exosome biogenesis pathway: pre-incubation with}

\section{B16-F10 cells}

Although some of the physicochemical approaches tested (mild sonication and thermal shock I) gave satisfactory results, we decided to test an alternative method in which the incorporation of NPs to exosomes would take place using the own cellular biogenesis mechanism. This had not been attempted before with therapeutic NPs capable of absorbing light in the NIR. Before harvesting any PEG-HGNs loaded exosomes (hereinafter termed exosPEG-HGNs), we studied the internalization of both HGNs and PEG-HGNs within B16F10 cells at different time points in order to find out the optimum configuration (with or without PEG coating) and the best incubation time to purify the final exosomes loaded with the NPs. Confocal microscopy, (Fig. 4B) revealed that, as expected from their large size, both kind of NPs were distributed in the cytoplasm rather than in the nuclei after $24 \mathrm{~h}$ of internalization. The amount of gold inside B16-F10 cells was quantified by Microwave Plasma-Atomic Emission Spectrometry (MP-AES) of the cellular pellet retrieved after incubation of the cells with AuNPs at different times (24 to $72 \mathrm{~h}$ ) and washing them to remove the supernatant fluid with any non-internalized NP (Fig. 4C). Both MP-AES results and confocal microscopy revealed that a more intense uptake of PEG-HGNs by B16-F10 cells compared to HGNs occurred at any of the times tested under the conditions used (Fig. 4B and $4 \mathrm{C}$ ). In particular, Fig. 4C indicates that an average uptake of $0.14 \mathrm{ng}$ of $\mathrm{Au}$ per cell was measured when PEG-HGNs were incubated for $24 \mathrm{~h}$ (corresponding to more than 600000 PEG-HGNs per cell). The higher internalization of PEG-coated particles compared to their uncoated counterparts has been reported previously for different cell lines (MSCs or tumoral cells) and attributed to a facilitated uptake thanks to the increase of NPs stability and reduced agglomeration provided by the PEG coating.45,46 
Considering the data provided by the internalization studies, we incubated cells with PEGHGNs during 24 hours in order to obtain exosPEG-HGNs. We carried out electronic microscopy studies to evaluate the presence or absence of NPs inside exosomes after their purification from B16-F10 cell cultures (Fig. 5). TEM images of purified exosPEG-HGNs show that a large proportion of exosomes secreted by parental cells were loaded with NPs (statistical analysis shown in Fig. 3B indicated that $49.11 \%$ of exosomes were loaded with AuNPs).

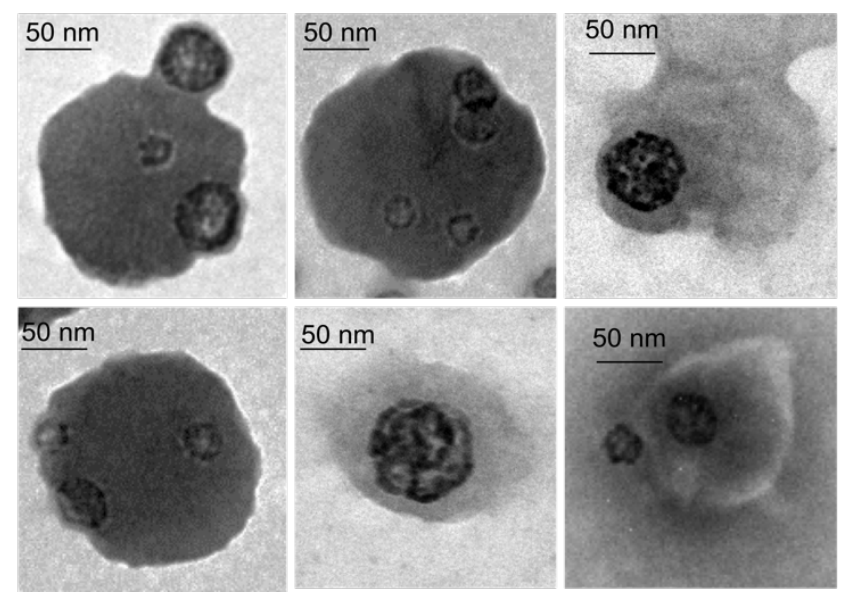

Figure 5. Representative TEM images obtained from exosomes released by B16-F10 after their incubation with PEG-HGNs during 24 hours.

To confirm that PEG-HGNs were inside exosomes and not attached to their surface, we carried out confocal microscopy studies to evaluate the co-localization between PEG-HGNs and B16-F10-exos. On the one hand, as a negative control, we added separately prepared NPs to a suspension of exosomes in PBS and they were directly observed under confocal microscopy. As Fig. 6A shows, segregated red spots (corresponding to reflection from aggregates of PEG-HGNs) and green spots (pertaining to exosomes labelled with PKH67 probe) were observed, with no apparent co-localization. On the other hand, when exosPEGHGNs purified from parental cells were visualized under confocal microscopy, yellow pixels 
could be observed, corresponding to the co-localization of the red spots from PEG-HGNsbased agglomerates reflection and the green fluorescence from exosomes.
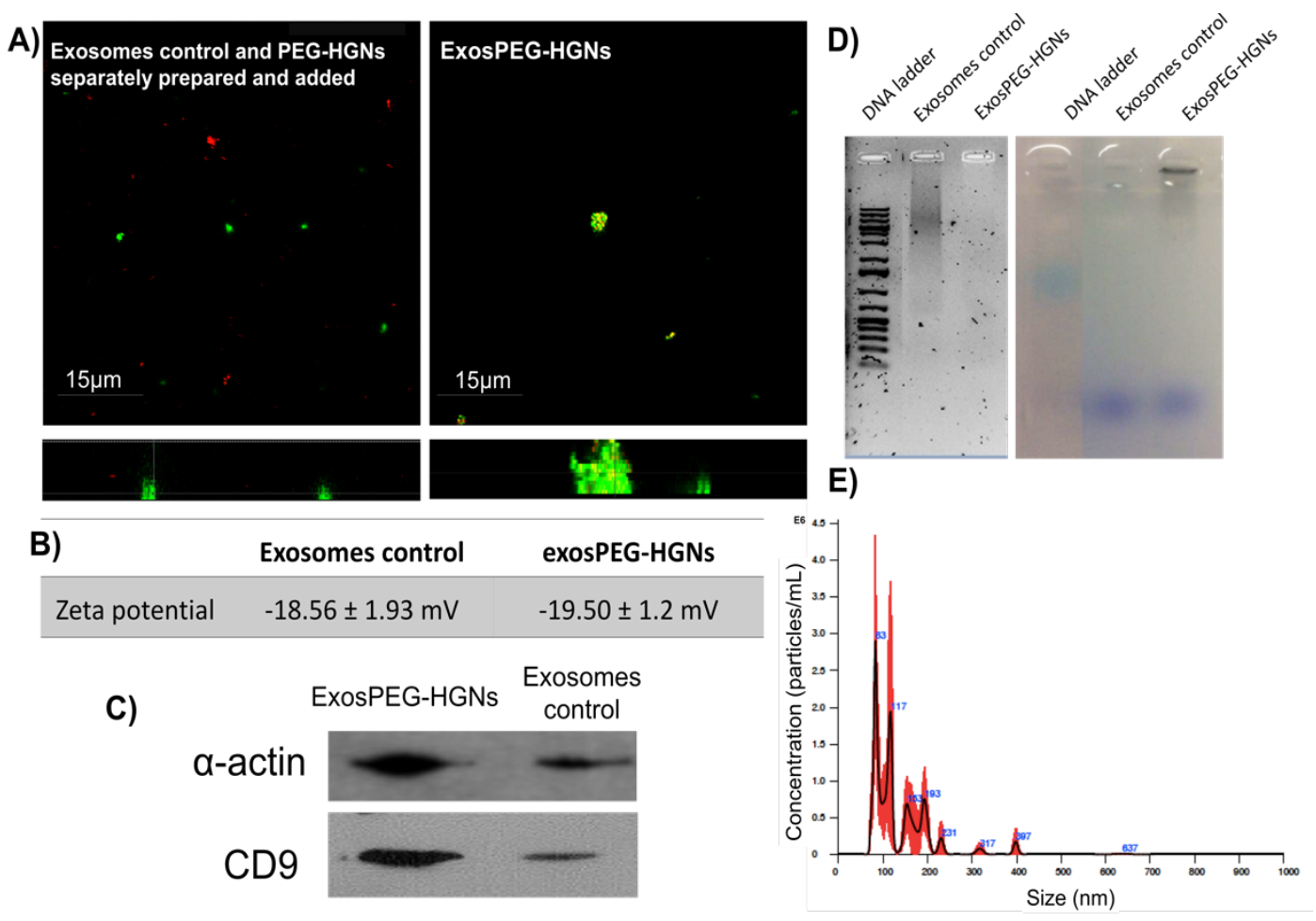

Figure 6. A) Co-localization study by confocal microscopy. Nanoparticle-based agglomerates were directly observed by reflection (red spots), and exosomes were labelled with PKH67 probe (green spots). Yellow pixels correspond with co-localization of exosomes and NPs. B) DLS measurements obtained of exosomes control and exosPEG-HGNs. C) Analysis of CD9 and $\alpha$-actin expression of empty and loaded exosomes by WB. D) Agarose retention assay for control (i.e. free from HGN inclusions) exosomes and exosPEG-HGNs. E) NTA diameter distribution obtained for exosPEG-HGNS.

A WB test of control exosomes and exosPEG-HGNs isolated from the same number of parental cells is shown in Fig. 6C. Clearly, specific exosomes proteins (CD9) were expressed in exosPEG-HGNs, confirming the exosomal nature of exosPEG-HGNs. Also, for the same number of secreting cells the expression levels of CD9 increased when PEG-HGNs were 
present, suggesting that PEG-HGNs could stimulate exosomal secretion. Similarly, expression of $\alpha$-actin (control protein) was elevated in exosomes secreted by B16-F10 cells exposed to PEG-HGNs. Additionally, control (HGN-free) exosomes and exosPEG-HGNs were subjected to an agarose retention assay. As it is well known that exosomes carry DNA with a large size $(>2.5 \mathrm{kbp}), 56$ after the formation of the complex exosome-nanoparticle the exosomal DNA gets retained in the well, as shown in Fig. 6D. The differences in migration rates cannot be explained by a larger size of the exosomes (mean diameters of empty or loaded exosomes obtained by NTA were similar, Fig. 2B and Fig. 6E, respectively) or by a change in their surface charge since, as shown in TEM and NTA results the diameter and the surface charge $(-18.56 \pm 1.93 \mathrm{mV}$ vs $-19.5 \pm 1.2 \mathrm{mV})$ of exosPEG-HGNs and control exosomes were rather similar. Betzer et al., observed that zeta potential of exosomes became slightly more negative due to the attachment of AuNPs to their surfaces.43 In our study, surface charge of exosome was not changed by the presence of the NPs confirming again the presence of the NPs inside the exosomes.

Only few studies have attempted the encapsulation of NPs within exosomes and most of them employ exogenous methods to achieve relatively low encapsulation efficiencies (table 1). The majority of the previous works tried to encapsulate SPIONs within exosomes by electroporation for MRI and tracking applications.37,38 Rather than encapsulating SPIONs, Qi et al. created a dual-functional exosome-based cluster by anchoring SPIONs on exosome surface through Tf-Tf receptor interaction for cancer therapy.41 However, to the best of our knowledge only three studies reported the encapsulation of AuNPs in exosomes. Alhasan et al. treated parental PC-3 cells with AuNPs of $13 \mathrm{~nm}$ conjugated to anti-miRNA21 to purify their exosomes.42 They observed that AuNPs were both localized inside exosomes and attached to their surface. In a different approach, Betzer et al. loaded exosomes with glucosecoated AuNPs from 5 to $20 \mathrm{~nm}$ in diameter. Although most of the AuNPs were successfully 
loaded within exosomes, few of them remained attached on the exosome surface.43 Finally, Roma-Rodrigues et al. functionalized AuNPs with thiolated oligonucleotides and incubated them with MCF-7 cells. Subsequently, they observed that $30 \%$ of the exosomes purified from parental cells were associated with AuNPs. They also incubated exosomes with AuNPs for $24 \mathrm{~h}$ achieving less than $5 \%$ of encapsulation. 44

The manipulation processes involved in those previous strategies decrease safety and increase the cost. Herein, we report a simple strategy to effectively load therapeutic PEGHGNs that absorb in the NIR region onto exosomes avoiding difficult or aggressive treatments (such as membrane disruption) or any chemical bonding on the exosomal membrane wall to preserve the natural characteristics of the produced exosomes. We have improved the encapsulation yield from $20 \%$ to $50 \%$ when parental cells were pre-treated with NPs before exosomes isolation. As previously shown, TEM and NTA results indicate that this loading approach is likely to preserve the natural size of the produced exosomes. We demonstrated the presence of AuNPs inside exosomes instead of attached to their surfaces using confocal microscopy co-localization assays and by zeta potential studies.

\section{PEG-HGNs are released through the exosomal secretory route}

To confirm that exosPEG-HGNs were in fact exosomes rather than other membrane derived vesicles, the implication of exosome biogenesis and release pathways in the exosPEG-HGNs production was evaluated. B16-F10 cells were pre-treated either with manumycin A or transfected with siRab27 to inhibit the budding of intraluminal vesicles into MVBs of the PEG-HGNs and to block the late steps of vesicle exocytosis in cells.57,58 Our results demonstrate that when exosome biogenesis of the B16-F10 cells was inhibited, the number of released exosPEG-HGNs was significantly lower. This fact, together with the previous characterization results, confirmed that exosPEG-HGNs were from endosomal origin. 
Exosome production was quantified by WB (Fig. 7) demonstrating that Tsg101 expression was lower after exosome genetic or chemical blockade in comparison with the level obtained for control B16-F10 cells. Again, Fig. S2 shows TEM images of both control and exosPEGHGNs purified from manumycin A and siRab27 treated cells. It clearly demonstrated that the number of exosomes produced by the untreated cells were significantly more abundant than the exosomes produced by the treated cells.

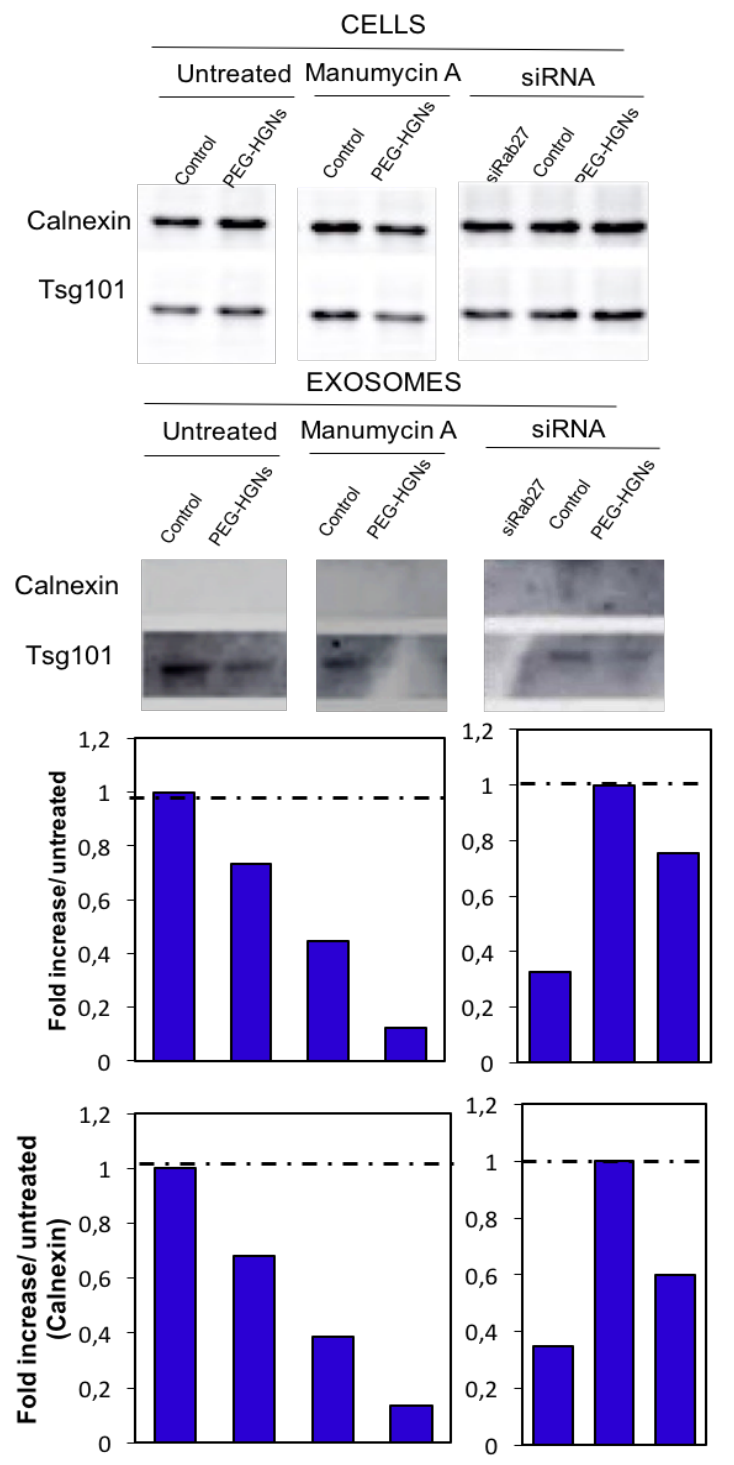

Figure 7. Characterization of exosomes produced by siRab27 and manumycin A treated cells. WB of calnexin (non-exosomal marker) and Tsg101 (specific exosome marker) of 
cellular and exosomal fractions. Images were analyzed using ImageJ software and quantified as a fold increase compared to untreated cell-derived exosomes.

\section{Theranostic properties of exosPEG-HGNs}

The combination of exosomes with NPs and in particular with gold nanoparticles as vectors for therapy and imaging of different physiological or pathological conditions has been described before. For instance, the use of the exosome-nanoparticles as a theranostic platforms is highlighted by Zhu et al. In fact, they established that exosomes play a crucial role in the NPs-induced system immune response by promoting the activation of the innate immune system (i.e., T-cells) and the maturation and differentiation of the dendritic cells.59 As a result, they took advantage of this phenomenon to develop a dual vaccine based on dendritic cells-derived exosomes against melanoma and lung tumors. The vesicle-based vaccine stimulated $\mathrm{T}$ lymphocytes and caused tumor rejection providing a highly effective and cell-free antitumor therapy based on exosomes. 60

Herein, in vitro optical hyperthermia experiments were performed to demonstrate the therapeutic potential of exosPEG-HGNs. The in vitro photothermal effect was analysed using $10 \mu \mathrm{g}$ of exosPEG-HGNs on B16-F10 cells. Figure 8A demonstrates that exosPEGHGNs were able to generate localized heat by the use of NIR light. Specifically, after 25 minutes of NIR laser exposure, a temperature increment in the culture media of $10.93+/-2.5$ ${ }^{\circ} \mathrm{C}$ and $6.5+/-1.21{ }^{\circ} \mathrm{C}$ were caused by the presence and absence of exosPEG-HGNs respectively. Similarly, the temperature increased $12.13+1.78{ }^{\circ} \mathrm{C}$ and $6.87+/-1.59^{\circ} \mathrm{C}$ in cultures incubated with or without exosPEG-HGNs after 30 minutes of NIR laser irradiation. Furthermore, Figure $8 \mathrm{~B}$ reveals that $808 \mathrm{~nm}$ irradiation did not reduce cell viability (measured by fluorescence microscopy) on cells treated with the laser without the exosPEGHGNs. On the contrary, the heat transmitted by this novel NIR-absorbing vector upon laser 
irradiation lead to tumor cell death in a cell culture in the presence of exosPEG-HGNs (a hole in the center of the well plate where the laser was applied was observed, corresponding with dead cells).

\begin{tabular}{|c|c|c|c|c|c|c|}
\hline Reference & Exosome origin & $\begin{array}{c}\text { Type of } \\
\text { nanoparticles }\end{array}$ & NPs size & Encapsulation method & Encapsulation efficacy & Reference \\
\hline Hood et al., 2014 & $\begin{array}{l}\text { Murine melanoma } \\
\text { cells }\end{array}$ & SPIONs & $5 \mathrm{~nm}$ & $\begin{array}{l}\text { Passive loading and } \\
\text { electroporation }\end{array}$ & $\begin{array}{l}\text { Iron concentration of SPION exosomes was from } \\
0.5 \text { to } 0.05 \mu \mathrm{g} \text { of iron per } \mu \mathrm{g} \text { exosome protein }\end{array}$ & 37 \\
\hline Hu et al., 2014 & $\begin{array}{l}\text { Murine melanoma } \\
\text { cells }\end{array}$ & SPIONs & $5 \mathrm{~nm}$ & Electroporation & $\begin{array}{l}\text { Iron concentration of SPION exosomes was } \\
\text { determined by optical density to be } \sim 0.5 \mu \mathrm{g} \text { iron } \\
\text { per } \mu \mathrm{g} \text { exosome protein }\end{array}$ & 38 \\
\hline Jia et al., 2018 & Raw264,7 cells & SPIONs & $5 \mathrm{~nm}$ & Electroporation & - & 62 \\
\hline $\begin{array}{l}\text { Altanerovaet al., } \\
2017\end{array}$ & $\begin{array}{l}\text { Mesenchymal } \\
\text { stem cells }\end{array}$ & Venofer & $65 \mathrm{~nm}$ & Labelling parental cells & - & 39 \\
\hline $\begin{array}{l}\text { Betzer et al., } \\
\quad 2017\end{array}$ & $\begin{array}{l}\text { Mesenchymal } \\
\text { stem cells }\end{array}$ & $\begin{array}{l}\text { Glucose-coated } \\
\text { AuNPs }\end{array}$ & from $5 \mathrm{~nm}$ to $20 \mathrm{~nm}$ & $\begin{array}{l}\text { Active loading (Internalized } \\
\text { and/or attached to the } \\
\text { external surface) }\end{array}$ & - & 43 \\
\hline $\begin{array}{l}\text { Roma-Rodrigues } \\
\text { et al., } 2017\end{array}$ & $\begin{array}{l}\text { Breast Carcinoma } \\
\text { Cells and normal } \\
\text { BTE cells }\end{array}$ & $\begin{array}{l}\text { AuNPs with } \\
\text { thiolted } \\
\text { oligonucleotides }\end{array}$ & $\begin{array}{c}\text { from } 14 \mathrm{~nm} \text { to } 30 \\
\mathrm{~nm}\end{array}$ & Labelling parental cells & $\begin{array}{l}30 \% \text { of AuNPs adsorbed or internalized within } \\
\text { exosomes }\end{array}$ & 44 \\
\hline Our work & $\begin{array}{l}\text { Murine melanoma } \\
\text { cells }\end{array}$ & PEG-HGNS & $40-45 \mathrm{~nm}$ & Labelling parental cells & $50 \%$ of exosomes loaded with PEG-HGNs & \\
\hline
\end{tabular}

Table 1. Strategies previously reported for the NPs encapsulation within exosomes

Related to the diagnostic/imaging potential of the vector, the inherent reflective optical properties of the exosPEG-HGNs allowed us to directly visualize clusters of exosPEGHGNs inside of the cells by reflection of the incident light from the confocal microscope at 488/490 nm ex/em (Figure 8C). Representative images from B16-F10 cells incubated with exosPEG-HGNs for 2, 4, 6, 8, 24 and $32 \mathrm{~h}$ clearly demonstrates the presence of exosPEGHGNs inside cell cytosol and highlights the potential use of this vector for in vitro cell labeling. Furthermore, the presence of the exosPEG-HGNs was semiquantified from confocal images. Figure 8D reveals that the presence of exosPEG-HGNs significantly increased inside the cells after $6 \mathrm{~h}$ of incubation and thus, the imaging properties of the exosomal vector. 

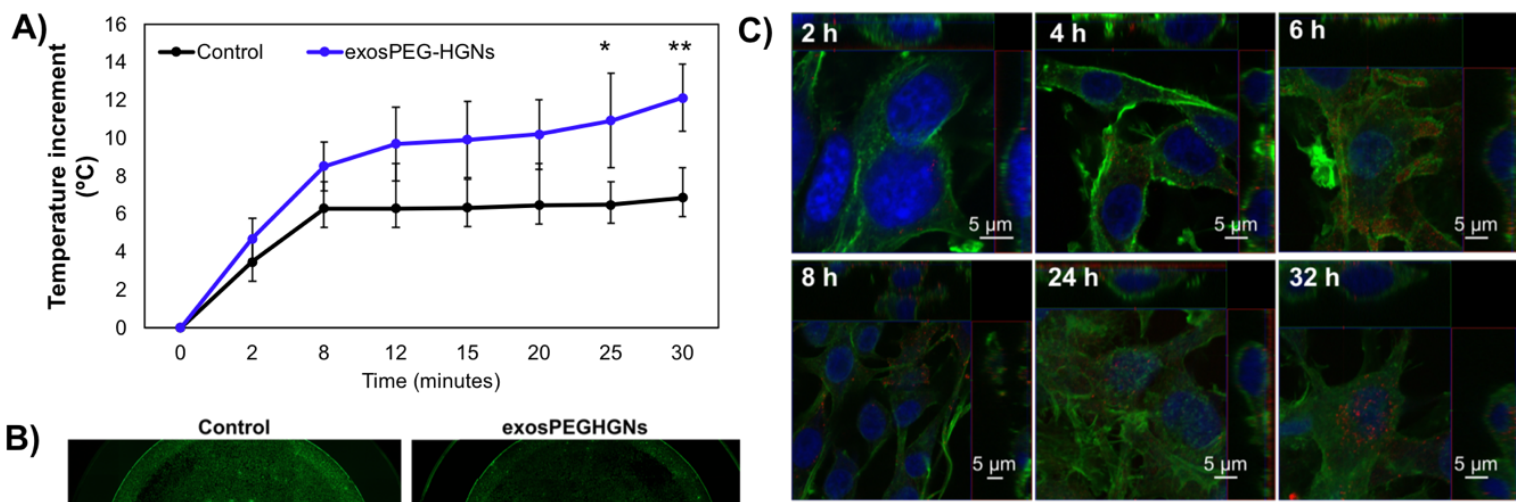

$24 \mathrm{~h}$

$32 \mathrm{~h}$

B)

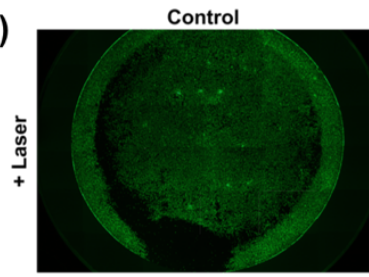

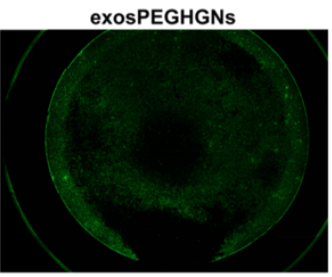

D) $\begin{array}{r}1,80 \mathrm{E}+07 \\ 1,60 \mathrm{E}+07\end{array}$

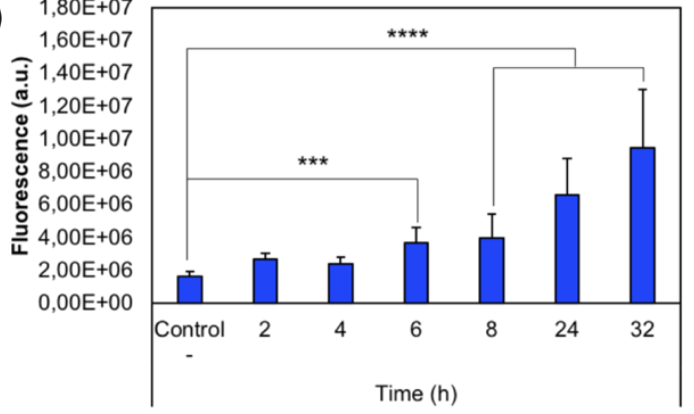

Figure 8. A) Temperature increase of cell culture after NIR laser irradiation in the presence or absence of exosPEG-HGNs. B) LIVE/DEAD images of B16-F10 irradiated or non irradiated and/or treated with exosPEG-HGNs. C) Merged confocal images of B16-F10 cells after treatment with exosPEG-HGNs for 2, 4, 6, 8, 24 and $32 \mathrm{~h}$. Actin fibers (phalloidin488) are shown in green, cell nuclei (Draq5) in blue and clusters of exosPEG-HGNs in red. D) Quantitative analysis of red fluorescent intensity in the cells at different timepoints. Error bars $\pm S D, n=3 . * * * P<0.001, * * * * P<0.0001$

\section{Materials and methods}

\section{Physicochemical methods for HGNs loading within exosomes}

AuNPs synthesis and exosome purification techniques are thoroughly described in the supplementary material section. All the physicochemical and biological techniques employed for NPs and vesicles characterization are also detailed in the supplementary material. HGNs internalization within B16-F10-exos was studied using different 
physicochemical methods. In all cases, exosomes were previously purified and then mixed with NPs. In a final volume of $200 \mu \mathrm{L}$ of PBS, $10 \mu \mathrm{g}$ of B16-F10-exos and $33 \mu \mathrm{g}$ of HGNs were put in contact and subjected to the processes described below. The methods were also applied to exosomes in the absence of NPs, to find out how all the employed techniques affected exosomes morphology. The encapsulation was defined as the percentage of exosomes containing NPs. The physicochemical strategies for NPs loading within exosomes were performed in triplicate.

\section{PEG-HGNs preincubation in B16-F10 cells and exosPEG-HGNs isolation}

Herein we developed an approach of exosome loading by incubating parental cells (B16-F10 cells) with HGNs or PEG-HGNs at subcytotoxic dose (see supplementary information) followed by isolation of the secreted PEG-HGNs loaded exosomes (exosPEG-HGNs) from the conditioned media. To develop an optimum procedure, we firstly evaluated the amount of NPs within cells at different time-points in order to choose the maximum internalization time for the isolation of exosPEG-HGNs. PEG-HGNs internalization within B16-F10 cells was evaluated by confocal microscopy and was quantified by MP-AES. Both procedures are detailed in the supplementary information section.

It is reported that parental cells can be loaded with exogenous compounds, which are then released into the conditioned media within exosomes.61 To obtain exosPEG-HGNs directly from cell cultures, NPs were added to ULTRACEN medium at the subcytotoxic dose $(0.125$ mg mL-1). B16-F10 cells were cultured with this enriched medium for $24 \mathrm{~h}$, allowing particles to be uptaken and incorporated into the biogenesis exosome pathway. Then, cells were strongly washed three times wih PBS to discard the non-internalized PEG-HGNs. Finally, the culture media was replaced by exosome-free medium during $48 \mathrm{~h}$ and the exosomes excreted during this period were purified from the supernatant by using 
ultracentrifugation cycles (described in the supplementary material section). The presence of PEG-HGNs in exosomes was analyzed by: TEM, co-localization with confocal microscopy, WB, Nanosight, Zeta potential and agarose gel electrophoresis retention assays. For TEM analysis, isolated PEG-HGNs-exos were deposited on Formvar carbon-coated grids to be further stained with phosphotungstic acid (3\%) and visualized as mentioned in the supplementary information section. In order to estimate the encapsulation efficiency, the number of exosomes loaded with at least one PEG-HGNs presented in TEM images were quantified and normalized by the total amount of exosomes presented in the sample. The experiments were performed at least in triplicate from independent cell cultures of B16-F10 cells.

For the co-localization studies, exosomes were labelled with PKH67 probe (Sigma-Aldrich, United States) as indicated by the manufacturer and PEG-HGNs were directly visualized by reflection. Samples were then visualized by confocal laser scanning microscopy in a $\mu$-Slide 8 Well (Ibidi, Germany) dissolved in PBS in $300 \mu \mathrm{L}$ of final volume (Spectral Confocal Microscope Leica TCA SP2). CD9 and $\alpha$-actin expression of control exosomes and exosPEG-HGNs were evaluated by $\mathrm{WB}$ as mentioned in the supplementary information section. Thakur et al., have previously reported that tumor derived exosomes carry double stranded DNA (dsDNA) larger than $2.5 \mathrm{~kb}$ associated with the outer membrane of exosomes.56 Thus, the PEG-HGNs loading within exosomes was also evaluated by detecting dsDNA of exosomes on an agarose gel electrophoresis retention assay. The loading buffer was mixed with the complexes before adding them into $1 \%$ of agarose gel containing SYBER ${ }^{\circledR}$ Safe stain (Life Technologies, United States). After running the gel at $150 \mathrm{~V}$ for 45 min, the migration of exosomal DNA was visualized in a UV chamber (Biorad ChemiDoc RXS, Unites States). The full procedures of Zeta potential and Nanosight measurements are shown in the supplementary information section. 


\section{Theranostic properties of exosPEG-HGNs}

B16-F10 cells were cultured at a cell density of 1.5 x 104 cells per well in a 48-well plate for $24 \mathrm{~h}$. Then, culture media was removed and $10 \mu \mathrm{g}$ of exosPEG-HGNs were added to the cells and the samples were irradiated with the NIR diode laser $(808 \mathrm{~nm}$, for $30 \mathrm{~min}$ at 1.5 $\mathrm{kW} \mathrm{cm-2).} \mathrm{After} \mathrm{that,} \mathrm{cells} \mathrm{were} \mathrm{incubated} \mathrm{with} \mathrm{the} \mathrm{LIVE/DEAD} \mathrm{kit} \mathrm{(ThermoFisher,} \mathrm{United}$ States) following manufacturer instructions and they were visualized under an inverted fluorescence microscope (Olympus IX81) performing a multi-alignment of 4x images to visualized the whole well. Control samples (not irradiated and/or not treated with exosPEGHGNs) were also evaluated to obtain the basal viability status of the cells.

B16-F10 cells were also seeded at a density of 2.5 x 104 cells per well onto $20 \mathrm{~mm}$ cover slips (in a 24-well plate) and incubated under culture conditions for 1 day. $10 \mu \mathrm{g}$ of exosPEGHGNs were then added to each well and they were incubated for 2, 4, 6, 8 and $24 \mathrm{~h}$. Cells were finally fixed, stained and visualized by confocal microscopy as mentioned in the supplementary information. Z-stack orthogonal projections were used to visualize the presence of exosomes inside cell cytosol.

\section{Conclusions}

The strategy herein developed proved to be highly efficient (around $50 \%$ encapsulation yield) to achieve the encapsulation into exosomes of plasmonic nanoparticles used in photothermal ablation (i.e., $45 \mathrm{~nm}$ PEG-HGNs). The protocol did not significantly alter their structure, morphology or expression of exosomal proteins. Interestingly, the PEG coating was key to the success of this procedure: internalization of uncoated HGNs was considerably less effective and therefore those NPs could not be incorporated in significant proportions into the exosome generation pathway. 
The complexes exosPEG-HGNs open the possibility to be used as theranostic vectors, as they are susceptible of producing extensive cell death upon NIR irradiation and of being visualized due to their inherent reflective optical properties. Given the outstanding properties of exosomes for stealth transport and cell targeting, the encapsulation of NIR-activable NPs opens up exciting possibilities in phototherapy.

\section{Conflicts of interest}

There are no conflicts to declare.

\section{$\underline{\text { Acknowledgements }}$}

We thank the ERC Consolidator Grant program (ERC-2013- CoG-614715, NANOHEDONISM) and the ERC Advanced Grant (ERC-2016-ADG-742684 CADENCE) for the financial support. CIBER-BBN and CIBER-CV are an initiative funded by the VI National R\&D\&i Plan 2008-2011 financed by the Instituto de Salud Carlos III with the assistance of the European Regional Development Fund. This was is partially funded by the Aragon Government (T57_17R p) cofounded by Feder 2014-2020 "Builing Europe from Aragon ". We thank the Scientific Facilities of Microscopy, Cytometry and Cell Culture (IACS) for their valuable help and continuous support. MS also acknowledge the Spanish Government for the receipt of a FPU predoctoral grant.

\section{$\underline{\text { References }}$}

1 H. Aldewachi, T. Chalati, M.N. Woodroofe, N. Bricklebank, B. Sharrack, P. Gardiner, Nanoscale, 2018, 10, 18.

2 M.U. Farooq, V. Novosad, E.A. Rozhkova, H. Wali, A.A. Fateh, P.B. Neogi, A. Neogi and Z. Wang, Sci Rep, 2018, 8, 1. 
Sebastián, M. Quintanilla, M. Arruebo, J. Santamaría and P. Marín-Duque, RSC Adv, 2016, $6,58723$.

4 N. Manohar, F.J. Reynoso, P.Diagaradjane, S. Krishnan and Cho S.H, Sci Rep, 2016, 6,1 .

5 C. Vidal, M. Tomás-Gamasa, P. Destito, F. López and J.L. Nat Commun, 2018, 9, 1.

$6 \quad$ L.A. Dykman and N.G. Khlebtsov, Acta Naturae, 2011, 3, 34.

7 V. Amendola, R. Pilot, M. Frasconi, O.M. Marago and Lati, J Phys Condens Matter, $2017,29,1$.

8 H. He, C. Xie and J. Ren, Anal Chem, 2008, 80, 5951.

9 P.-J.Debouttière, S. Roux, F. Vocanson, C. Billotey, O. Breuf, A. Favre-Reguillon, Y. Lin, S. Pellet-Rostaining, R. Lamartine, P. Perriat and O. Tillement, Adv Funct Mater. $200616,2330$.

10 R. Popovtzer, A. Agrawal, N.A. KotovA. A. Popovtzer, T.E. Carey, R. Kopelman, Nano Lett. 2008, 8, 4593.

11 Zhao W, Brook MA and Li Y, Nat Nanotechnol, 2008, 9, 2363.

12 G. Peng, U. Tisch, O. Adams, M. Hakim, N. Shehada, Y.Y. Broza, S. Billian, R. Abadh-Bornyak, A. Junten and H. Haick, Nat Nanotechnol, 2009, 4, 669.

13 A. Neely, C. Perry, B. Varisli, A.K. Singh, T. Arbneshi, D. Senapati, J.R. Kalluri and P.C. Ray, ACS Nano. 2009, 3, 2834.

14 S. Tang S and I. Hewlett, J Infect Dis, 2010, 201, S59.

15 X. Li, S.M. Robinson, A. Gupta, K. Saha, Z. Jiang, D.F. Moyano, A. Sahar, M.A. Riley and V.M. Rotello, ACS Nano. 2014, 8, 10682.

16 S.S. Agasti, A. Chompoosor, C. You, P. Ghosh, C.K. Kim and V.M. Rotello, J Am Chem Soc, 2009, 131, 5728. 
17 J. Xie, C. Xu, N. Kohler, Y. Hou and S. Sun, Adv Mater, 2007, 19, 3163.

18 C. Farokhzad, J. Cheng, B.A. Teply, I. Sherifi, S. Jon, P.W. Kantoff, J.P. Richie and R. Langer,PNAS, 2006, 103, 6315.

19 S. Wilhelm, A.J. Tavares, Q. Dai, S. Ohta, J. Audet, H.F. Dvorak and W.C.W. Chan, Nat Rev Mater, 2016, 1, 1.

20 T. Skotland, K. Sandvig and A. Llorente, Prog Lipid Res, 2017, 66, 30.

21 R.W. Yeo, R.C. Lai, B. Zhang, S.S. Tan, Y. Yin, B.J. The, S.K. Lim, Adv Drug Deliv Rev, 2013, 65, 336.

22 G. Raposo and W.J. Stoorvogel,Cell Biol, 2013, 200, 373.

23 N.P. Hessvik and A. Llorente, Cell Mol Life Sci, 2018, 75,193.

24 F. Shahabipour, N. Barati, T.P. Johnston, G. Derosa, P. Maffioli, A. Sahebkar, J Cell Physiol, 2017, 232, 1660.

25 C. Kahlert C and P. Kalluri, J Mol Med, 2013, 91, 431.

26 G. Lachenal, K. Pernet-Gallay, M. Chivet, F.J. Hemming, A. Belly, G. Bodon, B. Blot, G. Haase, Y. Goldberg and R. Sadoul, Mol Cell Neurosci, 2011, 46, 409.

27 T. Xiao, W. Zhang, B. Jiao, C. Pan, X. Liu and L. Shen, Transl Neurodegener. $2017,6,1$.

28 N. Amabile, P.E. Rautou, A. Tedgui and C.M. Boulanger, Semin Thromb Hemost, $2010,36,907$.

29 S. Rana, S. Yue, D. Stadel, M. Zöller, Int J Biochem Cell Biol. 2012, 44, 1574.

30 A. Kalani, A. Tyagi and N. Tyagi, Mol Neurobiol, 2014, 49,590.

31 X. Zhuang, X. Xiang, W. Grizzle, D. Sun, S. Zhang, R.C. Axtell, S. Ju, J. Mu, L. Zhang, L. Steinman, S. Miller and H-G. Zhang, Mol Ther, 2009, 19, 1769.

32 M.J. Haney, N.L. Klyachko, Y. Zhao, R. Gupta, E.G. Plotnikava, Z. He, A. Piroyan, M. Sokolsky, A.V. Kabanov and E.V. Batrakova, J Control Release, 2016, 21, 18. 
Biotechnol, 2011, 29, 341.

34 S.I. Ohno, M. Takanashi, K. Sudo, S. Ueda, A. Ishikawa, N. Matsuyama, K. Fujita,

T. Mizutani, T. Ohgi, T. Ochiya, N. Gotoh and M. Kuroda, Mol Ther, 2013, 21, 185.

35 Y. Tian, S. Li, J. Song, T. Ji, M. Zhu, G.J. Anderson, J. Wei and G. Nie, Biomaterials. 2014, 35, 2383.

36 T. Yang, P. Martin, B. Fogarty, A. Brown, K. Schurman, R. Phipps, V.P. Yin, P. Lockman and S.Bai, Pharm Res, 2015, 32, 2003.

37 J.L. Hood, M.J. Scott and S.A Wickline, Anal Biochem, 2014, 448, 41.

38 L. Hu, S.A. Wickline and J.L. Hood, Magn Reson Me,. 2015, 74, 266.

39 U. Altanerova, M. Babincova, P. Babinec, K. Benejova, J. Jakubechova, V. altanerova, M. Zduriencikova, V. Repiska and C. Altaner, Int J Nanomedicine, 2017, 12, 7923.

40 A. Busato, R. Bonafede, P. Bontempi, I. Scambi, L. Schiaffino, D. Benati, M. Malatesta, A. Sbarbati, P. Marzola and R. Mariotti, Curr Protoc Cell Biol, 2017, 75, 3.44 .15

41 H. Qi, C. Liu, L. Long, Y. Ren, S. Zhang, X. Chang, X. Qian, H. Jie, J. Zhao, J. Sun, X. Hou, X Yuan and C. Kang, ACS Nano, 2016, 10, 3323.

42 A.H. Alhasan, P.C. Patel, C.H.J. Choi and C.A. Mirkin, Small. 2014, 10, 186.

43 O. Betzer, N. Perets, A. Angel, M. Motiei, T. Sadan, G. Yadid, D. Offen and R. Popovtzer, ACS Nano, 2017, 11, 10883.

44 C. Roma-rodrigues, F. Pereira, A.P. Alves de Matos, M. Fernandes, P.V. Baptista, A.R. Fernandes, Nanomedicine, 2017, 13, 1389.

45 M. M. Encabo-Berzosa, M. Sancho-Albero, A. Crespo, V. Andreu, V. Sebastián, S. Irusta, M. Arruebo, P. Martín-Duque and J. Santamaría, Nanoscale, 2017, 9, 9848. 
Martín-Duque and J. Santamaría, J Nanobiotechnology. 2019, 17, 1.

47 G.T. Bardi, M.A. Smith, J.L. Hood, Cytokine. 2018, 105, 63.

48 J.L. Hood, S. San Roman and S.A. Wickline SA, Cancer Res. 2011, 71, 63.

49 T. Akagi, K. KatoN. Hanamura, M. Kobayashi and T. Ichiki, Japanese J Appl Physocs. 2014, 53, 6S.

50 C. Théry, K.W. Witwer, E. Aikawa et al., J. Extracell Vesicles, 2018,7,1.

51 D.G. Mazzucchelli, N.A. Cellier, V. Mshviladzade, R. Elias, Y-H. Shim, D.

Touboul, L. Wuinton, A. Brunelle, O. Laprévote, E.A. De Pauw and M-C.A. De PauwGillet, J Proteome Res, 2008, 7, 1683.

52 J. Wahlgren, T.D. Karlson, M. Brisslert, F.V. Sani, E. Telemo, P. Sunnerhagen and H. Valadi, Nuclei Acids Res, 2012, 40, e130.

53 M.S. Kim, M.J. Haney, Y. Zhao, V. Mahajan, I. Deygen, N.L. Klyachko, E. Inskoe, A. Piroyan, M. Sokolsky, O. Okolie, S.D. Hingtgen, A.V. Kabanov and E.V. Batrakova, Nanomedicine, 12, 655.

54 X. Luan, K. Sansanaphongpricha, I. Myers, H. Chen, H. Yuan and D. Sun, Acta Pharmacol Sin., 2017, 38, 754.

55 M.L. Prieto, Ö. Oralkan, B.T. Khuri-Yakub, M.C. Maduke, Plos One,2013, 8, 1. 56 B.K. Thakur, H. Zhang, A. Becker, I. Matei, Y. Huang, B. Costa-Silva, Y. Zheng, A. Hoshino, H. Brazier, J. Xiang, C. Williams, R. Rodriguez-Barrueco, J.M. Silva, W. Zhang, S. Hearn, O. Elemento, N. Paknejad, K. Manova-Todorova, K. Welte, J. Bromberg, H. Peinado and D. Lyden. Cell Res. 2014, 24, 766.

57 A. Datta, H. Kim, Lal M, L. McGee, A. Johnson, A.A. Moustafa, J.C. Jones, D. Mondal, M. Ferrer and A.B. Adbel-Mageed, Cancer Lett, 2017, 408, 73. 
58 M. Ostrowski, N.B. Carmo, S. Krumeich, I. Fanget, G. Raposo, A. Savina, C.F. Moita, K. Schauer, A.N. Hume, R.P. Fraitas, B. Goud, P. Benaroch, N. Hacohen, M.

Fakuda, C. Desnos, M.C. Seabra, F. Darchen, S. Amigorena, L.F. Moita and C. Thery, Nat Cell Biol, 2009,12, 19.

59 M. Zhu, X. Tian, X. Song, Y. Li, Y. Tian, Y. Zhao and G. Nie, Small, 2012, 8, 2841.

60 X.Tian, M. Zhu, Y. Tian, G. A. Ramm, Y. Zhao and G. Nie, Biomaterials, 2012, $33,6147$.

61 L. Pascucci, V. Coccè, A. Bonomi, D. Ami, P. Ceccaralli, E. Ciusani, L. Viganò, A. Locatelli, F. Sisto, S.M. Soglia, E. Parati, M.E. Bernardo, M. Muraca, G. Alessandri, G. Bondiolotti and A. Pessina. J Control Release, 2014, 192, 262.

G. Jia, Y. Han, Y. An, Y. Ding, C. He, X. Wang and Q. Tang, Biomaterials, 2018, 178, 30. 


\title{
Supplementary information
}

\section{Efficient encapsulation of large theragnostic nanoparticles in cell-derived} exosomes: Leveraging the exosomal biogenesis pathway to obtain hollow

\section{gold nanoparticle-exosome hybrids}

\author{
María Sancho-Albero ${ }^{a, b}$, Dr. Marimar Encabo-Berzosa ${ }^{a, b}$, Manuel Beltrán-Visiedo ${ }^{a, b}$, Dr. Lola \\ Fernández-Messina ${ }^{c, d}$, Dr. Víctor Sebastián ${ }^{a, b}$, Prof. Francisco Sánchez-Madrid ${ }^{c, d}$, Dr. Manuel \\ Arruebo $^{a, b, *}$, Prof. Jesús Santamaría, ${ }^{a, b}$ and Dr. Pilar Martín-Duque $e^{e, f}$
}

a Department of Chemical Engineering, Aragón Institute of Nanoscience (INA). Univeristy of Zaragoza, Campus Rio Ebro-Edificio I+D+I, C/ Mariano Esquillor S/N, 50018- Zaragoza-Spain.

b Networking Research Center of Bioengineering, biomaterials and Nanomedicine, CIBER-BBN, 28029Madrid, Spain

c Servicio de Inmunología, Instituto de Investigación Sanitaria Princesa (IP), Hospital Universitario de la Princesa, Universidad Autónoma de Madrid. C/ Diego de León 62, 28006-Madrid, Spain.

d Centro de Investigación Biomédica en Red de Enfermedades Cardiovasculares (CIBER- CV), Av. Monforte de Lemos 3-5, 28029-Madrid, Spain.

e Fundación Araid, 500009-Zaragoza, Spain.

f Instituto Aragonés de Ciencias de la Salud (IACS/IICS Aragón), Centro de Investigación Biomédica de Aargón (CIBA), Zaragoza, Spain.

\section{Corresponding author}

Dr Manuel Arruebo and Prof. Jesús Santamaría

Department of Chemical Engineering

Aragon Nanoscience Institute

C/Mariano Esquillor s/n

Zaragoza University

50018 Zaragoza, Spain

Tel: $+34876555437+3487655545311$

Email: arruebom@unizar.es, jesus.santamaria@unizar.es 


\section{Materials and methods}

\subsection{Synthesis and characterization of $H G N S$}

All chemicals used in the HGNs synthesis were obtained from Sigma-Aldrich: cobalt (II) chloride hexahydrate, sodium citrate tribasic dehydrate, poly (vinylpyrrolidone) (PVP) $\mathrm{Mw}=55000 \mathrm{Da}$, gold (III) chloride hydrate (50\% purity), sodium borohydride, and poly (ethylene glycol)-ether thiol (PEG $1000 \mathrm{Da} \mathrm{Mw}$ ). HGNs synthesis was scaled from the previously reported protocol reported by Preciado-Flores et al.1 The resulted HGNs were functionalized with monofunctional poly (ethylene glycol)-ether thiol (PEG $1000 \mathrm{Da} \mathrm{Mw}$ ). After and before the PEGylation step, NPs were characterized by transmission electron microscopy (T20-FEI Tecnai transmission electron microscopy (TEM)), UV-VIS spectroscopy and measurement of surface charge. ImageJ software (NIH-RSB) was employed to determine the average particle diameter of both HGNs and PEG-HGNs, measuring a minimum of 200 particles.

\subsection{Cell culture conditions}

Metastatic murine skin melanoma cells were provided by cell services from Cancer Research-UK and cultured in Dubecco's modified Eagle's medium (DMEM; Biowest, France) supplemented with $10 \%$ FBS (GIBCO, United States), $1 \%$ penicillin/streptomycin and $1 \%$ amphotericin (Biowest, France), under normoxic conditions. Ultracentrifugated (ULTRACEN medium, $100000 \mathrm{~g}, 8 \mathrm{~h}, 4^{\circ} \mathrm{C}$ ) serum was used in order to guarantee exosomes free medium.

\subsection{Exosome isolation and characterization}

B16-F10-exos were isolated following a protocol previously developed in our lab and based on successive ultracentrifugation cycles from cell culture supernatants2. First of all, cells were cultured until confluence. In the first step, supernatants were collected and were 
centrifuged for $20 \mathrm{~min}$ at $2000 \mathrm{~g}$ at $4{ }^{\circ} \mathrm{C}$. Secondly, another centrifugation step was carried out for $1 \mathrm{~h}$ at $10000 \mathrm{~g}$ at $4{ }^{\circ} \mathrm{C}$ to eliminate the microvesicles. To obtain the exosomal fraction, the samples were ultracentrifuged for $2 \mathrm{~h}$ at $100000 \mathrm{~g}$ at $4{ }^{\circ} \mathrm{C}$. However, another ultracentrifugation step in the same conditions was necessary in order to eliminate the surrounded proteins of the exosome. The obtained precipitates were resuspended in PBS and a Pierce BCA protein assay (Thermo Fisher Scientific, United States) was performed in order to estimate the protein content in the exosomes sample. B16-F10-exos size, shape and morphology were characterized by TEM by contrasting them with phosphotungstic acid (3 $\%)$. From TEM images, the average diameter of exosomes was obtained using Image J software (NIH-RSB). The surface charge of exosomes was also determined at $\mathrm{pH}=7$ in PBS (Brookhaven 90 plus and ZetaPALS software). To identify specific surface proteins such as CD9, western blotting was carried out. Briefly, $25 \mu \mathrm{g}$ of B16-F10-exos were precipitated with acetone $(1: 1 \mathrm{w} / \mathrm{w})$, lysed in Laemmli buffer (Sigma-Aldrich, United States) and boiled at $95^{\circ} \mathrm{C}$ during 5 min. Subsequently, proteins were separated by $12 \%$ SDS-polyacrylamide gel electrophoresis during $2 \mathrm{~h}$ and transferred to nitrocellulose membranes at $4^{\circ} \mathrm{C}$ during 4 h. The membranes were blocked overnight with non-fat dry milk in tris-buffered saline (TBS) $5 \%$. After that, blots were incubated with primary antibodies CD9, 1:2000 (Abcam, United Kingdom), TSG101, 1:500 (Abcam, United Kingdom), calnexin, 1:1000 (Abcam, United Kingdom) and $\alpha$-actin, 1:10000 (Sigma-Aldrich, United States). Membranes were washed three times with TBS-Tween (TBST) followed by the incubation of the secondary antibody (Sigma-Aldrich, United States). Finally, membranes were extensively washed and after the addition of chemiluminescence substrate, imaging was carried out. Cellular lysates from parental cells were used as negative control. Size distribution of exosomes was measured using Nanosight (Malvern Instruments, United Kingdom). Samples were diluted 
in PBS to optimize the number of particles in the field of view. They were measured at room temperature in triplicate for 60 seconds.

\subsection{Physicochemical methods for HGNs-loaded exosomes}

HGNs internalization within B16-F10-exos was studied using different physicochemical methods.

Incubation at room temperature. HGNs internalization within exosomes at room temperature was performed by mixing them during $18 \mathrm{~h}$ without stirring.

Incubation at room temperature with saponin. It is well known that saponin selectively interacts with membrane cholesterol molecules, forming pores and/or removing cholesterols from the cell membrane.3 Once B16-F10-exos were mixed with HGNs, saponin was added at $0.2 \%$ and the sample was mixed under stirring during $20 \mathrm{~min}$ at room temperature.

Thermal shock $I$. In this method, the exosome-NPs mixture was frozen at $-80{ }^{\circ} \mathrm{C}$ during 30 min and subsequently allowed to defrost. This freeze-thaw cycle was developed three times.

Thermal shock II. A classical strategy for bacterial transformation was applied to the encapsulation of HGNs within exosomes4. In this case, the sample was heated at $42{ }^{\circ} \mathrm{C}$ for 30 seconds, followed by incubating it at $4{ }^{\circ} \mathrm{C}$ during 2 minutes.

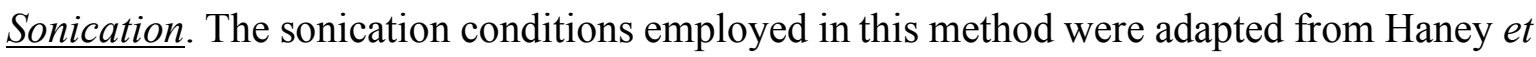
al., employing ultrasounds higher than $20 \mathrm{KHz}$ to generate transitory pores in exosomal membranes.5 Exosome-NP mixture was sonicated (UW 2200 Bandelin Sonopuls, Germany) in an ice bath at $500 \mathrm{~V}, 2 \mathrm{kHz}$ during 6 cycles ( 4 seconds of pulse and 2 seconds of pause).

Electroporation. The conditions previously developed by Hu et al. for the electroporation of exosomes to introduce SPIONs were employed when following this method.6 Briefly, 
suspended exosomes were electroporated in the presence of HGNs (Genepulser Xcell electroporator, Biorad, United Kingdom) in $4 \mathrm{~mm}$ path length electroporation cuvette. A single pulse was applied under a $950 \mu \mathrm{F}$ of capacitance and infinite resistance.

\subsection{Cytotoxicity evaluation}

To determine cell viability of B16-F10 cells under the effect of HGNs and PEG-HGNs, the Blue Cell Viability assay ${ }^{\circledR}$ (Promega, United States) was employed as previously reported.7 The experiment was performed in triplicate.

\subsection{PEG-HGNs preincubation in B16-F10 cells and exosPEG-HGNs isolation}

The cellular uptake was observed under a confocal microscope (Spectral Confocal Microscope Leica TCA SP2 and ZEISS LSM880 Confocal Laser Scanning Microscope) with a $63 x$ oil immersed N.A. 1.40 objective. Cells were seeded at a density of $2 \cdot 104$ cells onto $20 \mathrm{~mm}$ cover slips (in a 24-well plate) and cultured for $24 \mathrm{~h}$. The NPs $(0.125 \mathrm{mg} \mathrm{mL}$ 1) resuspended in DMEM were added to the cells and incubated for $24 \mathrm{~h}$. Then, cells were fixed with para-formaldehyde (PFA) $4 \%$. Cytoplasmic actin was labeled by staining with phalloidin-Alexa488 (Invitrogen, United States) and Draq-5 was used to observe the nuclei. Reflection of the incident light at $488 / 490 \mathrm{~nm}$ was used to directly visualized HGNs and PEG-HGNs-based agglomerates. Z-stack orthogonal projections were analyzed to determine the presence of NPs inside the cytosol.

Finally, to quantify the amount of gold inside B16-F10 cells, they were seeded onto 6-well plates at a density of $2.5 \times 105$ cells per well and were grown for $24 \mathrm{~h}$. Later, HGNs and PEG-HGNs dispersions $(0.125 \mathrm{mg} \mathrm{mL}-1)$ were prepared in fresh DMEM and added to the cell cultures for 24, 48 and $72 \mathrm{~h}$. After these time points, cells were harvested and washed twice with PBS (1500 rpm, $5 \mathrm{~min}$ ). Control samples (cells without treatment) were collected using the same protocol. The cellular pellets obtained were digested with $10 \%$ Aqua regia 
$(\mathrm{HNO} 3+3 \mathrm{HCl})$ in $1.5 \mathrm{~mL}$ of $\mathrm{dH} 2 \mathrm{O}$. Digestion was performed at room temperature for $1 \mathrm{~h}$. Total amount of gold derived from HGNs and PEG-HGNs was determined by MP-AES (4100 MP-AES, Agilent Technologies, United States). Furthermore, considering NPs as hollow spheres of known diameter and thickness and knowing gold metal density, the gold mass present in each particle (HGH and PEG-HGN) could be estimated, and from this, the number of NPs per cell at 24, 48 and $72 \mathrm{~h}$ was calculated after allowing for the contribution of PEG mass, obtained from the thermogravimetric analysis (TGA). 6

\subsection{PEG-HGNs are released through the exosomal secretory route}

To evaluate the exosome biogenesis and release pathways, 2 x 105 cells per well were seeded onto a 6-well plate and were grown for 24 hours in complete DMEM medium. Then PEGHGNs were added at $0.125 \mathrm{mg} \mathrm{mL}-1$ to the cell cultures for another 24 hours. Before purifying exosPEG-HGNs, B16-F10 cells were treated during 2 hours with $2 \mu \mathrm{M}$ manumycin A (Sigma-Aldrich, United States) in order to suppress exosome secretion. It is reported that manumycin A inhibits endocytosis via inhibition of Ras signaling in cancer cells.57 Separately, cells were transfected with $250 \mathrm{nM}$ ON-TARGET plus mouse Rab27a siRNA-SMART pool (Dharmacon, United States) using Lipofectamine 2000 (ThermoFisher, United States). Rab27 is a GTPase that mediates the late steps of vesicle exocytosis in cells.58 Thereafter, DMEM medium was replaced by ULTRACEN medium during $48 \mathrm{~h}$ for the subsequent exosome isolation procedure. In order to compare the number of exosomes secreted when cells were treated with manumycin A or when they were transfected with Rab27-siRNA, exosPEG-HGNs produced by untreated B16-F10 cells were considered as controls. ExosPEG-HGNs isolated from the different treated cells, were characterized by TEM, WB and BCA as previously described the exosome isolation and characterization section. From TEM images a semiquantitative analysis was performed in 
order to estimate the number of empty or loaded exosomes produced by treated cells compared with the control cells. In WB, calnexin and TSG101 expression were analysed as shown in the supplementary information section.

\subsection{Statistical analysis}

All the results expressed as the mean \pm the standard deviation were performed in triplicate. The statistical analysis of the data was carried out using the GraphPad Prism 7.04. Significance was determined by one-way analysis of variance (ANOVA) using the Tukey's multiple comparisons test for the encapsulation strategies, cytotoxicity and MP-AES results and the Dunnett's multiple comparison test for the MP-AES data: $* P<0.05, * * P<0.01$, $* * * P<0.001, * * * * P<0.0001$.

\section{Results and discussion}

\section{Physicochemical characterization of HGNS}

Both HGNs showed a diameter of $36.3 \pm 5.4 \mathrm{~nm}$ and PEG-HGNs had a PEG shell of around $5 \mathrm{~nm}$ (Fig. 1A). As shown in Fig. 1B, both NPs dispersions presented a characteristic surface plasmon resonance peak above $800 \mathrm{~nm}$ (NIR region), making them useful for optical hyperthermia applications (this peak is characteristic of spherical PEG-HGNs of approximately $40 \mathrm{~nm}$ ). Remarkably, it is crucial to obtain NPs of this size and morphology to guarantee their potential application as optical hyperthermia absorbing moieties in the NIR region.8 Z-potential measurements gave a negative surface charge in HGNs $(-16.38 \pm$ $2.72 \mathrm{mV})$ and PEG-HGNs $(-16.56 \pm 2.96 \mathrm{mV})$ at $\mathrm{pH} 7$.

\section{$\underline{\text { B16-F10-exos characterization }}$}

B16-F10-exos were spherical vesicles with a diameter of $95.1 \pm 27.8 \mathrm{~nm}$ (Fig 2A) as expected from the literature.9,10 They presented a double lipidic membrane with a thickness 
of almost $10 \mathrm{~nm}$ (observed in TEM images from Fig 2A). Exosome diameter was also confirmed by NTA, revealing also a diameter of $159.7 \pm 57.4 \mathrm{~nm}$ (Fig. 2B). Exosomes were negatively charged (zeta potential of $-18.56 \pm 1.93 \mathrm{mV}$ in $10 \mathrm{mM} \mathrm{KCl}$ at $\mathrm{pH}$ 7) due to the phospholipid nature of the exosomal membranes.11 Fig. $2 \mathrm{C}$ shows the results of the Western Blot (WB) assays indicating that B16-F10-exos expressed a housekeeping gene ( $\alpha$ actin), an exosomal membrane protein (CD9) and a citoplasmatic exosomal marker (TSG101). On the contrary, when using a non-exosomal protein (calnexin) no band was observed. These results confirm that B16-F10-exos correspond with exosomes rather than with other extracellular vesicles of different nature. 12

\section{References}

62 S. Preciado-Flores, D. Wang, D.A. Wheeler, R. Newhouse, J.K. Hensel, A. Schwartzberg, L. Wang, J. Zhu, M. Barboza-Flores and J.Z. Zhang, J. Mater. Chem., 2011, 21, 2344.

63 M. Sancho-albero, N. Navascués, G. Mendoza, V. Sebastián, M. Arruebo, P. Martín-Duque and J. Santamaría, J Nanobiotechnology. 2019, 17, 1.

64 M. Wassler, I. Jonasson, R. Persson and E. Fries, Biochem J., 1987, 247,207.

65 M. Rahimzadeh, M. Sadeghizadeh, F. Najafi, S. Arab and H. Mobasheri, Mol Biol Res Commun., 2016, 5, 257.

66 M.J. Haney, N.L. Klyachko, Y. Zhao, R. Gupta, E.G. Plotnikova, Z. He, T. Patel, A. Piroyan, M. Sokolsky, A.V. Kabanov and E.V. Batrakova, J Control Release., 2015, 207, 18.

67 L. Hu, S.A. Wickline and J.L. Hood, Magn Reson Med. 2015, 74, 266. 
68 M. M. Encabo-Berzosa, M. Sancho-Albero, A. Crespo, V. Andreu, V. Sebastián, S. Irusta, M. Arruebo, P. Martín-Duque and J. Santamaría, Nanoscale, 2017, 9, 9848.

69 M.M. Encabo-berzosa, M. Gimeno, L. Lujan, M. Sancho-Albero, L. Gómez, V. Sebastián, M. Quintanilla, M. Arruebo, J. Santamaría and P. Marín-Duque, RSC $A d v, 2016,6,58723$.

70 G.T. Bardi, M.A. Smith, J.L. Hood, Cytokine. 2018, 105, 63.

71 J.L. Hood, S. San Roman and S.A. Wickline SA, Cancer Res. 2011, 71, 63.

72 T. Akagi, K. KatoN. Hanamura, M. Kobayashi and T. Ichiki, Japanese J Appl Physocs. 2014, 53, 6S.

73 C. Théry, K.W. Witwer, E. Aikawa et al., J. Extracell Vesicles, 2018,7,1. 


\section{SUPPLEMENTARY FIGURES}

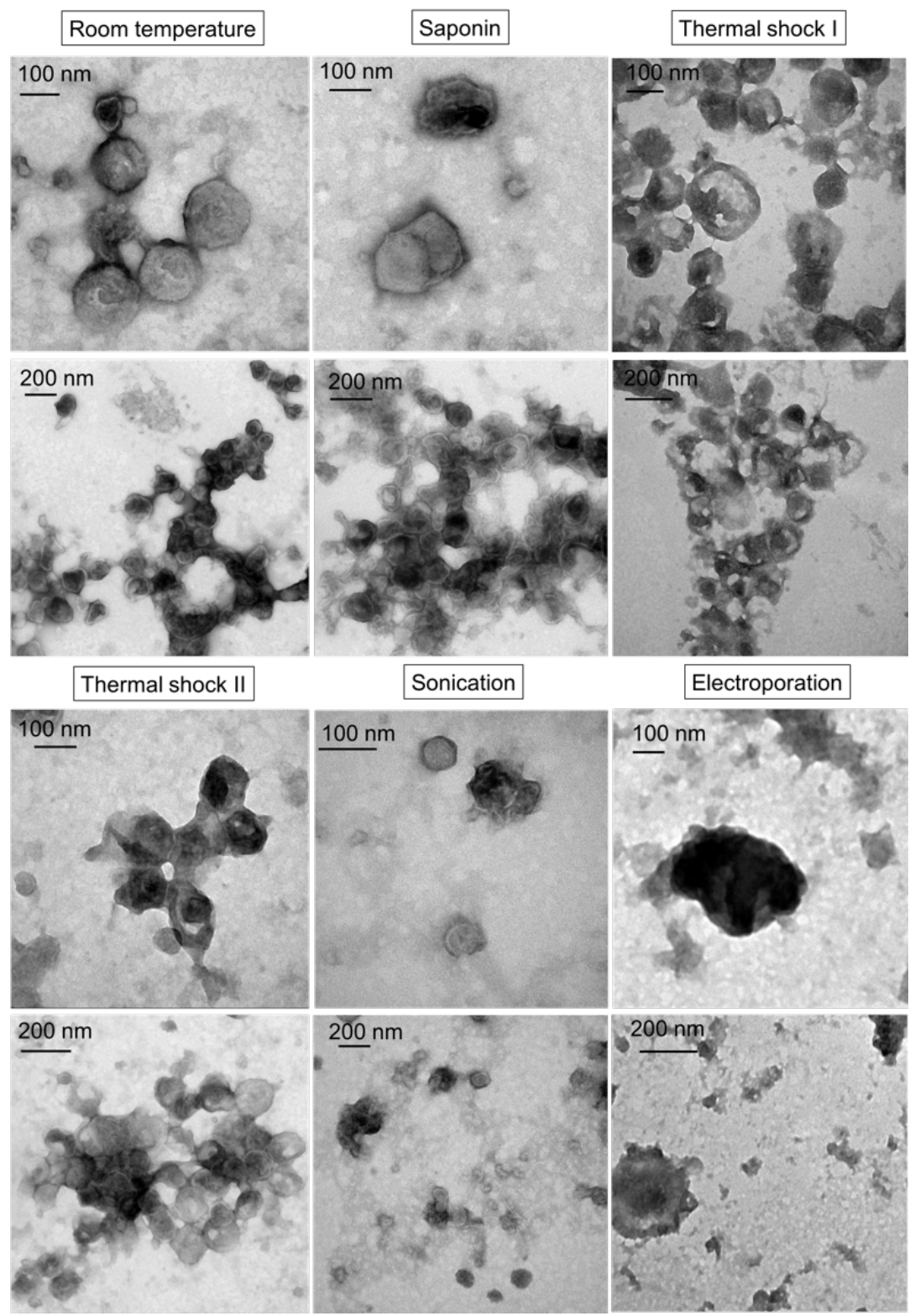

Figure S1. TEM images of exosomes treated by the different physicochemical approaches in absence of NPs (incubation at room temperature with the presence or absence of saponin, two different thermal shock procedures, sonication and electroporation). 


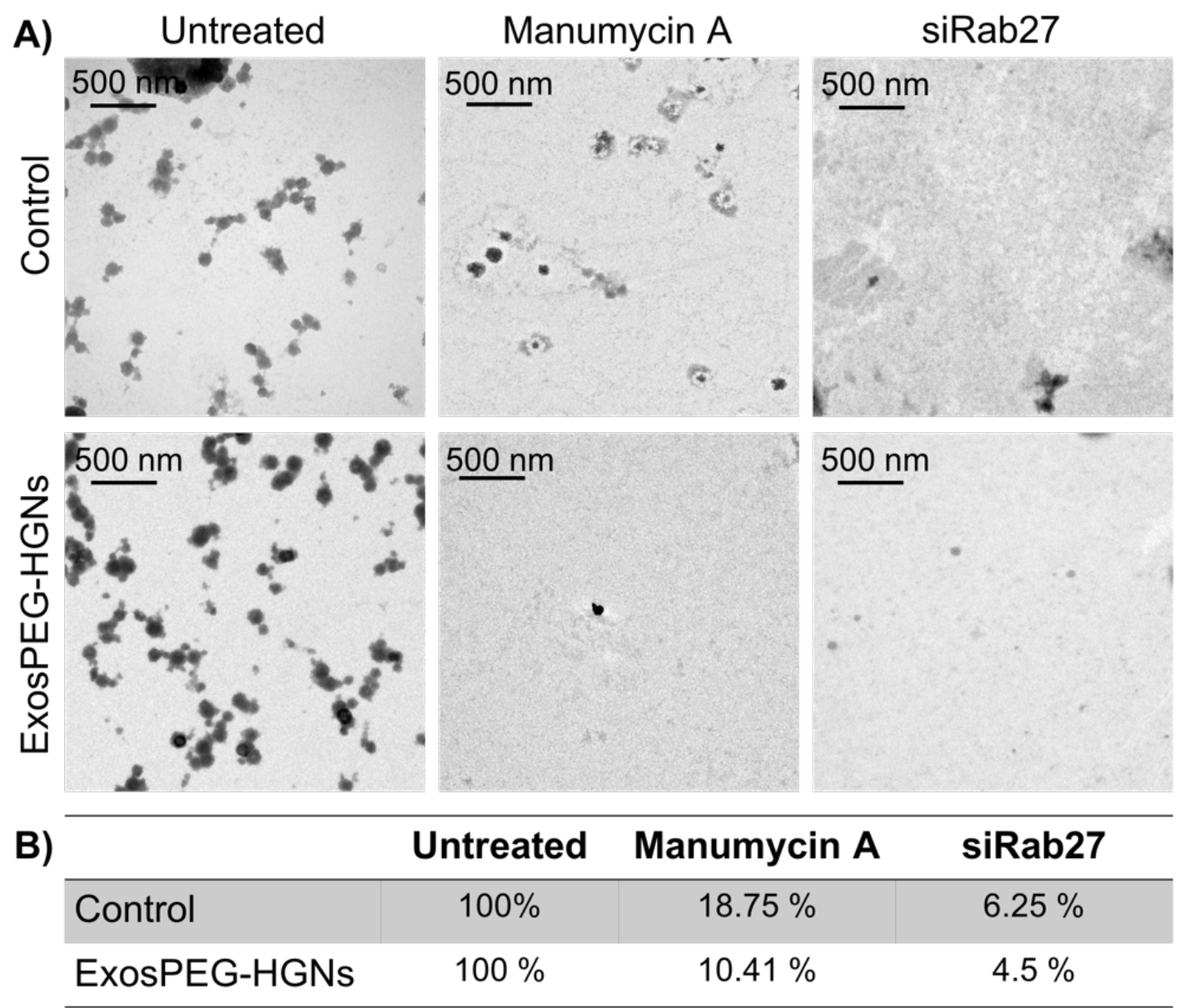

Fig. S2. A) Representative TEM images obtained from exosomes released by untreated B16F10 cells, by manumycin A treated cells or by siRab27 transfected cells. B) Semiquantitative analysis of secreted exosomes released by untreated B16-F10 cells, by manumycin A treated cells from TEM images. 


\section{SUPPLEMENTARY TABLES}

\begin{tabular}{|c|c|c|}
\hline & Summary & Adjusted P Value \\
\hline Incubation R.T. with saponin (0.2 \%) vs. Incubation R.T. & ns & 0.9988 \\
\hline Incubation R.T. with saponin $(0.2 \%)$ vs. Thermal shock I & ns & 0.9241 \\
\hline Incubation R.T. with saponin $(0.2 \%)$ vs. Thermal shock II & ns & 0.573 \\
\hline Incubation R.T. with saponin (0.2 \%) vs. Sonication & ns & 0.9645 \\
\hline Incubation R.T. with saponin (0.2 \%) vs. Electroporation & * & 0.0124 \\
\hline Incubation R.T. with saponin (0.2 \%) vs. Incubation with B16-F10cells & $* * * *$ & $<0.0001$ \\
\hline Incubation R.T. vs. Thermal shock I & ns & 0.7093 \\
\hline Incubation R.T. vs. Thermal shock II & $\mathrm{ns}$ & 0.8343 \\
\hline Incubation R.T. vs. Sonication & ns & 0.7993 \\
\hline Incubation R.T. vs. Electroporation & * & 0.0296 \\
\hline Incubation R.T. vs. Incubation with B16-F10cells & $* * * *$ & $<0.0001$ \\
\hline Thermal shock I vs. Thermal shock II & ns & 0.1276 \\
\hline Thermal shock I vs. Sonication & ns & $>0.9999$ \\
\hline Thermal shock I vs. Electroporation & $* *$ & 0.0018 \\
\hline Thermal shock I vs. Incubation with B16-F10cells & $* * * *$ & $<0.0001$ \\
\hline Thermal shock II vs. Sonication & ns & 0.168 \\
\hline Thermal shock II vs. Electroporation & ns & 0.2706 \\
\hline Thermal shock II vs. Incubation with B16-F10cells & $* * * *$ & $<0.0001$ \\
\hline Sonication vs. Electroporation & $* *$ & 0.0024 \\
\hline Sonication vs. Incubation with B16-F10cells & $* * * *$ & $<0.0001$ \\
\hline Electroporation vs. Incubation with B16-F10cells & $* * * *$ & $<0.0001$ \\
\hline
\end{tabular}

Table S1. Statistical analysis of the encapsulation of NPs within exosomes. 


\begin{tabular}{|c|c|c|}
\hline HGNs $24 \mathrm{~h}$ & Summary & Adjusted P Value \\
\hline Control vs. $0.5 \mathrm{mg} / \mathrm{mL}$ & $* * * *$ & $<0.0001$ \\
\hline Control vs. $0.250 \mathrm{mg} / \mathrm{mL}$ & $* *$ & 0.0024 \\
\hline Control vs. $0.125 \mathrm{mg} / \mathrm{mL}$ & $\mathrm{ns}$ & 0.355 \\
\hline Control vs. $0.06 \mathrm{mg} / \mathrm{mL}$ & ns & 0.8128 \\
\hline Control vs. $0.03 \mathrm{mg} / \mathrm{mL}$ & $\mathrm{ns}$ & 0.917 \\
\hline Control vs. $0.015 \mathrm{mg} / \mathrm{mL}$ & $\mathrm{ns}$ & 0.8727 \\
\hline Control vs. $0.006 \mathrm{mg} / \mathrm{mL}$ & $\mathrm{ns}$ & 0.6999 \\
\hline PEG-HGNs $24 \mathrm{~h}$ & Summary & Adjusted P Value \\
\hline Control vs. $0.5 \mathrm{mg} / \mathrm{mL}$ & $\mathrm{ns}$ & 0.0813 \\
\hline Control vs. $0.250 \mathrm{mg} / \mathrm{mL}$ & $\mathrm{ns}$ & 0.6189 \\
\hline Control vs. $0.125 \mathrm{mg} / \mathrm{mL}$ & $\mathrm{ns}$ & 0.2749 \\
\hline Control vs. $0.06 \mathrm{mg} / \mathrm{mL}$ & $\mathrm{ns}$ & 0.3113 \\
\hline Control vs. $0.03 \mathrm{mg} / \mathrm{mL}$ & ns & 0.8098 \\
\hline Control vs. $0.015 \mathrm{mg} / \mathrm{mL}$ & $\mathrm{ns}$ & 0.8757 \\
\hline Control vs. $0.006 \mathrm{mg} / \mathrm{mL}$ & ns & 0.6958 \\
\hline HGNs $48 \mathrm{~h}$ & Summary & Adjusted P Value \\
\hline Control vs. $0.5 \mathrm{mg} / \mathrm{mL}$ & $* * *$ & 0.0007 \\
\hline Control vs. $0.250 \mathrm{mg} / \mathrm{mL}$ & $* * *$ & 0.0005 \\
\hline Control vs. $0.125 \mathrm{mg} / \mathrm{mL}$ & $\mathrm{ns}$ & 0.1041 \\
\hline
\end{tabular}




\begin{tabular}{|c|c|c|}
\hline Control vs. $0.06 \mathrm{mg} / \mathrm{mL}$ & $\mathrm{ns}$ & 0.695 \\
\hline Control vs. $0.03 \mathrm{mg} / \mathrm{mL}$ & $\mathrm{ns}$ & 0.5105 \\
\hline Control vs. $0.015 \mathrm{mg} / \mathrm{mL}$ & $\mathrm{ns}$ & 0.6599 \\
\hline Control vs. $0.006 \mathrm{mg} / \mathrm{mL}$ & $\mathrm{ns}$ & 0.1819 \\
\hline PEG-HGNs $48 \mathrm{~h}$ & Summary & Adjusted P Value \\
\hline Control vs. $0.5 \mathrm{mg} / \mathrm{mL}$ & $* * *$ & 0.0007 \\
\hline Control vs. $0.250 \mathrm{mg} / \mathrm{mL}$ & $* *$ & 0.0049 \\
\hline Control vs. $0.125 \mathrm{mg} / \mathrm{mL}$ & ns & 0.0824 \\
\hline Control vs. $0.06 \mathrm{mg} / \mathrm{mL}$ & $\mathrm{ns}$ & 0.206 \\
\hline Control vs. $0.03 \mathrm{mg} / \mathrm{mL}$ & ns & 0.9997 \\
\hline Control vs. $0.015 \mathrm{mg} / \mathrm{mL}$ & ns & 0.9997 \\
\hline Control vs. $0.006 \mathrm{mg} / \mathrm{mL}$ & $\mathrm{ns}$ & 0.9995 \\
\hline HGNs $72 \mathrm{~h}$ & Summary & Adjusted P Value \\
\hline Control vs. $0.5 \mathrm{mg} / \mathrm{mL}$ & $* * *$ & 0.0007 \\
\hline Control vs. $0.250 \mathrm{mg} / \mathrm{mL}$ & $* *$ & 0.0049 \\
\hline Control vs. $0.125 \mathrm{mg} / \mathrm{mL}$ & ns & 0.0824 \\
\hline Control vs. $0.06 \mathrm{mg} / \mathrm{mL}$ & ns & 0.206 \\
\hline Control vs. $0.03 \mathrm{mg} / \mathrm{mL}$ & ns & 0.9997 \\
\hline Control vs. $0.015 \mathrm{mg} / \mathrm{mL}$ & ns & 0.9997 \\
\hline Control vs. $0.006 \mathrm{mg} / \mathrm{mL}$ & ns & 0.9995 \\
\hline
\end{tabular}




\begin{tabular}{|c|c|c|}
\hline PEG-HGNs 72 h & Summary & Adjusted P Value \\
\hline Control vs. $0.5 \mathrm{mg} / \mathrm{mL}$ & $\mathrm{ns}$ & 0.622 \\
\hline Control vs. $0.250 \mathrm{mg} / \mathrm{mL}$ & $\mathrm{ns}$ & 0.711 \\
\hline Control vs. $0.125 \mathrm{mg} / \mathrm{mL}$ & $\mathrm{ns}$ & 0.9882 \\
\hline Control vs. $0.06 \mathrm{mg} / \mathrm{mL}$ & $\mathrm{ns}$ & 0.992 \\
\hline Control vs. $0.03 \mathrm{mg} / \mathrm{mL}$ & $\mathrm{ns}$ & $>0.9999$ \\
\hline Control vs. $0.015 \mathrm{mg} / \mathrm{mL}$ & $\mathrm{ns}$ & $>0.9999$ \\
\hline Control vs. $0.006 \mathrm{mg} / \mathrm{mL}$ & $\mathrm{ns}$ & 0.9995 \\
\hline
\end{tabular}

Table S2. Statistical analysis of the cytotoxicity experiment. 


\begin{tabular}{|l|c|c|}
\cline { 2 - 3 } \multicolumn{1}{c|}{ A) } & Summary & $\begin{array}{c}\text { Adjusted P } \\
\text { Value }\end{array}$ \\
\hline Control vs. PEG-HGNs 24 h & $* * * *$ & $<0.0001$ \\
\hline Control vs. PEG-HGNs 48 h & ns & 0.9925 \\
\hline Control vs. PEG-HGNs 72 h & ns & 0.8895 \\
\hline Control vs. HGNs 24 h & ns & 0.9999 \\
\hline Control vs. HGNs 48 h & ns & 0.9999 \\
\hline Control vs. HGNs 72 h & ns & 0.9999 \\
\hline
\end{tabular}

\begin{tabular}{|c|c|c|}
\hline В) & Summary & $\begin{array}{c}\text { Adjusted P } \\
\text { Value }\end{array}$ \\
\hline PEG-HGNs $24 \mathrm{~h}$ vs. PEG-HGNs $48 \mathrm{~h}$ & $* *$ & 0.0018 \\
\hline PEG-HGNs $24 \mathrm{~h}$ vs. PEG-HGNs $72 \mathrm{~h}$ & $* *$ & 0.0029 \\
\hline \multirow[t]{2}{*}{ PEG-HGNs $48 \mathrm{~h}$ vs. PEG-HGNs $72 \mathrm{~h}$} & ns & 0.9363 \\
\hline & Summary & $\begin{array}{c}\text { Adjusted P } \\
\text { Value }\end{array}$ \\
\hline HGNs 24 h vs. HGNs 48 h & ns & 0.1067 \\
\hline HGNs $24 \mathrm{~h}$ vs. HGNs $72 \mathrm{~h}$ & ns & 0.6576 \\
\hline HGNs 48 h vs. HGNs 72 h & ns & 0.5691 \\
\hline
\end{tabular}

Table S3. Statistical analysis of the MP-AES experiment. A) Comparison of all the conditions with the untreated cells. B) Comparison of cells treated with one type of NPs at 24, 48 and 72 hwith every other samples treated with these NPs at other time points 


\begin{tabular}{|c|c|c|}
\cline { 2 - 3 } \multicolumn{1}{c|}{} & Summary & Adjusted P Value \\
\hline $0 \mathrm{~min}$ & $\mathrm{~ns}$ & $>0.9999$ \\
\hline $2 \mathrm{~min}$ & $\mathrm{~ns}$ & 0.9745 \\
\hline $8 \mathrm{~min}$ & $\mathrm{~ns}$ & 0.5989 \\
\hline $12 \mathrm{~min}$ & $\mathrm{~ns}$ & 0.1282 \\
\hline $15 \mathrm{~min}$ & $\mathrm{~ns}$ & 0.0915 \\
\hline $20 \mathrm{~min}$ & $\mathrm{~ns}$ & 0.0726 \\
\hline $25 \mathrm{~min}$ & $*$ & 0.0197 \\
\hline $30 \mathrm{~min}$ & $* *$ & 0.0037 \\
\hline
\end{tabular}

Table S4. Statistical analysis of cell culture temperatures after NIR laser irradiation. 


\begin{tabular}{|c|c|c|}
\hline & Summary & $\begin{array}{c}\text { Adjusted P } \\
\text { Value }\end{array}$ \\
\hline Control vs. $2 \mathrm{~h}$ & $\mathrm{~ns}$ & 0,2762 \\
\hline Control vs. $4 \mathrm{~h}$ & $\mathrm{~ns}$ & 0,6152 \\
\hline Control vs. $6 \mathrm{~h}$ & $* * *$ & 0,0003 \\
\hline Control vs. $8 \mathrm{~h}$ & $* * * *$ & $<0,0001$ \\
\hline Control vs. $24 \mathrm{~h}$ & $* * * *$ & $<0,0001$ \\
\hline Control vs. $48 \mathrm{~h}$ & $* * * *$ & $<0,0001$ \\
\hline
\end{tabular}

Table S5. Statistical analysis of exosPEG-HGNs inside cell cytoplasm from confocal images. 